hep-th/0701173

\title{
Towards Minkowski Vacua in Type II String Compactifications
}

\author{
Andrei Micu ${ }^{1 \dagger, \ddagger}, \quad$ Eran Palti ${ }^{2 \S}, \quad$ Gianmassimo Tasinato ${ }^{2 \Phi}$ \\ ${ }^{1}$ Physikalisches Institut der Universität Bonn \\ Nussallee 12, D-53115, Bonn, Germany \\ ${ }^{2}$ Rudulf Peierls centre for Theoretical Physics, University of Oxford \\ Keble Road, Oxford, UK.
}

\begin{abstract}
We study the vacuum structure of compactifications of type II string theories on orientifolds with $S U(3) \times S U(3)$ structure. We argue that generalised geometry enables us to treat these non-geometric compactifications using a supergravity analysis in a way very similar to geometric compactifications. We find supersymmetric Minkowski vacua with all the moduli stabilised at weak string coupling and all the tadpole conditions satisfied. Generically the value of the moduli fields in the vacuum is parametrically controlled and can be taken to arbitrarily large values.
\end{abstract}

\footnotetext{
${ }^{\dagger}$ email: amicu@th.physik.uni-bonn.de

${ }^{\ddagger}$ On leave from IFIN-HH Bucharest

$\S$ email: palti@thphys.ox.ac.uk

๑email: tasinato@thphys.ox.ac.uk
} 


\section{Introduction}

Flux compactifications constitute a promising direction to look for string vacua with stabilised moduli [1, 2]. However, most of the supersymmetric solutions found so far feature a vacuum with negative cosmological constant, while Minkowski vacua with all the moduli stabilised in a perturbative regime have remained elusive 1 .

On the other hand, in the last years it became clear that the original Calabi-Yau compactifications (even with fluxes turned on) represent only a fraction of all the possibilities which lead to supersymmetric theories in lower dimensions. In particular, in Refs. [3, 4] it was shown that supersymmetric ground states in four dimensions are related to internal manifolds with $S U(3) \times S U(3)$ structure which are called twisted generalised Calabi-Yau manifolds.

Another important idea that allows to extend the class of manifolds suitable for compactification is that of duality. For example, it is well established that type IIA/IIB string theories are related by T-duality/mirror symmetry, in the absence of fluxes. By insisting that this remains true when fluxes are turned on, we should be able to find new configurations that are the geometric dual of fluxes. In particular, it is expected that the NS-NS fluxes play a major role, as it is well known that T-duality mixes the metric with the $B$-field: this was indeed confirmed in Refs. [7, 8, 9] where it was shown that the mirror/T -dual of the electric NS-NS fluxes are manifolds with torsion. These were half-flat manifolds, of which twisted-tori are a subclass. Compactifications on these type of manifolds was consequently studied in [9]-28] It was realised that these type of manifolds induced superpotentials that had vacua where all the moduli were stabilised, all be it in an anti-deSitter vacuum.

The question of what type of manifolds are needed to recover the mirror in the presence of magnetic NS fluxes however remained a little more obscured. It was realised that these compactifications are not geometric in the usual sense of geometry Such non-geometric manifolds were studied in [5, 8, [29] [54 and the mirror to the magnetic NS fluxes were termed nongeometric fluxes. It was also conjectured in [14] that possible compactifications that would lead to the mirrors of magnetic NS fluxes are compactifications on manifolds described by generalised geometry. This was made more precise in [30, 31].

In this paper we study compactifications of type IIA and IIB string theories on (nongeometric) orientifolds with $S U(3) \times S U(3)$ structure with the purpose of finding four-dimensional supersymmetric Minkowski solutions. We argue that the formalism of generalised geometry allows us to treat non-geometric compactifications in a way very similar to geometric compactifications. Indeed by generalising the derivative operator to a covariant derivative for T-dualities we show that we are able to derive (a subclass of) the four-dimensional superpotentials of [49, 55] from a compactification. Within this formalism we are able to derive the superpotential for an arbitrary number of moduli. This is a crucial step toward finding Minkowski vacua. We show that, under mild constraints on the numbers of moduli, the superpotential we consider exhibits a rich spectrum of Minkowski vacua with all the moduli stabilised and tadpole conditions satisfied.

The paper outline is as follows. In section 2 we introduce some concepts in generalised geometry and describe how we deal with the non-geometric nature of the compactifications. In section 3 we derive the superpotentials and tadpole constraints for compactifications of type

\footnotetext{
${ }^{1}$ For a recent world-sheet approach for models with no Kähler moduli at strong coupling see [5]. Also see [6] for a non-perturbative possibility.
} 
IIA and type IIB string theory on orientifolds with $S U(3) \times S U(3)$ structure. In section 4 we study the vacuum structure of the superpotential for a number of simple cases and show that generically the vacuum spectrum can include Minkowski vacua with all the moduli stabilised at parametrically controlled values.

\section{Some generalised geometry}

In this section we give a brief overview of the parts of generalised geometry that are relevant for this work. We use the term 'generalised geometry' to denote both generalised complex geometry and generalised almost complex geometry. Generalised geometry has been developed by mathematicians [56, 57, 58, 59, 60, 61, 62 and almost in parallel applied to Physics. By now there are a number of excellent reviews for physicists [1, 29, 31, 63] which we follow for some of this section. In the first part of this section we introduce the objects and notations that we use in this paper. Following this we discuss an extension to the formalism in the form of a new derivative operator that can be thought of as gauging transformations of generalised complex structures. This kind of derivative has also been recently proposed in [31. We end the section with a discussion of the some of the physics associated with generalised geometry.

\subsection{Generalised complex geometry}

Generalised complex geometry is the generalisation of complex geometry to $T \oplus T^{*}$, the tangent and cotangent bundles. An element of $T \oplus T^{*}$ is in general a sum of a vector $X$ and a one-form $\xi$. It is useful to write quantities in terms of matrices and vectors where the rows and columns denote whether the elements are in $T$ or in $T^{*}$. Then a generalised almost complex structure can be defined as a map $\mathcal{J}: T \oplus T^{*} \rightarrow T \oplus T^{*}$ that squares to $-\mathbb{1}_{2 d}$, where $d$ is the real dimension of the manifold, and satisfies a Hermiticity condition $\mathcal{J}^{T} \mathcal{I} \mathcal{J}=\mathcal{I}$ where

$$
\mathcal{I}=\left(\begin{array}{cc}
0 & \mathbb{1}_{d} \\
\mathbb{1}_{d} & 0
\end{array}\right)
$$

An example of a generalised almost complex structure is one induced by an almost complex structure $I$

$$
\mathcal{J}_{I}=\left(\begin{array}{cc}
I & 0 \\
0 & -I^{T}
\end{array}\right) .
$$

Another example is one that is induced by an almost symplectic two-form $J$

$$
\mathcal{J}_{J}=\left(\begin{array}{cc}
0 & -J^{-1} \\
J & 0
\end{array}\right) .
$$

In general, a generalised almost complex structure will be some combination of the two. Given a generalised almost complex structure it is possible to generate a new one by a $B$-transformation defined as

$$
\mathcal{J}_{B}=\left(\begin{array}{ll}
1 & 0 \\
B & 1
\end{array}\right) \mathcal{J}\left(\begin{array}{cc}
1 & 0 \\
-B & 1
\end{array}\right),
$$

where $B$ is a real two-form, $B \in \Lambda^{2} T^{*}$. Similarly we can also generate a new one through a $\beta$-transform

$$
\mathcal{J}_{\beta}=\left(\begin{array}{cc}
1 & \beta \\
0 & 1
\end{array}\right) \mathcal{J}\left(\begin{array}{cc}
1 & -\beta \\
0 & 1
\end{array}\right) \text {, }
$$


where $\beta$ is a bivector, $\beta \in \Lambda^{2} T$. Given two generalised almost complex structures, $\mathcal{J}_{a}$ and $\mathcal{J}_{b}$ that are compatible (ie they commute) we also have a positive definite generalised metric

$$
\mathcal{G}=-\mathcal{J}_{a} \mathcal{J}_{b}
$$

The two generalised almost complex structures reduce the structure group of the metric from $O(d, d)$ to $U\left(\frac{d}{2}\right) \times U\left(\frac{d}{2}\right)$.

Consider a a generalised complex structure $\mathcal{J}$. Then since $\mathcal{J}^{2}=-\mathbb{1}$ we can define projectors $\frac{1}{2}(1 \pm i \mathcal{J})$ that split $T \oplus T^{*}$ into two subspaces $L$ and $\bar{L}$ that correspond to the $+i$ and $-i$ eigenvalues of $\mathcal{J}$. The subspace $L$ is maximal isotropic. Isotropic means that for any $v, w \in L$ we have

$$
\langle v, w\rangle=0,
$$

where $\langle\ldots, \ldots\rangle$ is the natural bracket on $T \oplus T^{*}$ defined as

$$
\langle X+\xi, Y+\eta\rangle=\frac{1}{2}(\xi(Y)+\eta(X))
$$

for general elements $X+\xi \in T \oplus T^{*}$. Maximal refers to the dimension of the subspace being the maximum value on the manifold, which is the dimension of the manifold. Then for each $\mathcal{J}$ there is a unique splitting into $L$ and $\bar{L}$ and conversely, a generalised complex structure is equivalent to a maximal isotropic subspace $L \subset\left(T \oplus T^{*}\right) \otimes \mathbb{C}$ such that $L \cap \bar{L}=\{0\}$. The type, $k$, of a maximal isotropic is the co-dimension of its projection onto $T$. Note that $B$-transforms do not change the type. In fact all the maximal isotropic subspaces are related by $B$-transforms. $\beta$-transforms can change the type by an even number.

\subsection{Pure spinors and $S U(3) \times S U(3)$ structure}

The generalised almost complex structure defined in the previous section can also be expressed in terms of a pure spinor $\Phi$. This comes from the fact that the subspace $L$, of annihilators of a pure spinor $\Phi$

$$
(X+\xi) \Phi=0, \quad X+\xi \in L,
$$

is exactly a maximal isotropic subspace. The spinor can in turn be thought of as a formal sum of forms of even or odd degrees (corresponding to pure spinors with positive and negative chirality respectively). This is just the generalisation of generating a sum of forms from a spinor by acting on it with gamma matrices. In terms of the spinor $B$-transforms and $\beta$-transforms act as $\Phi \rightarrow e^{B} \Phi$ and $\Phi \rightarrow e^{\beta} \Phi$ respectively. Just as the existence of two generalised almost complex structures reduced the metric structure group to $U\left(\frac{d}{2}\right) \times U\left(\frac{d}{2}\right)$, the existence of two compatible (commuting) non-vanishing pure spinors reduces the structure group to $S U\left(\frac{d}{2}\right) \times S U\left(\frac{d}{2}\right)$ where the extra reduction comes from the non-vanishing constraint.

In this paper we are concerned with compactifications to four dimensions and so require a structure group $S U(3) \times S U(3)$. Since each spinor also provides a supersymmetry parameter we see that compactifications on manifolds with $S U(3) \times S U(3)$ structure lead to $\mathcal{N}=2$ supersymmetry in four dimensions.

It is possible to prove [60] that a pure spinor must always take the form

$$
\Phi=e^{B+i J} \wedge \Omega_{k},
$$


where $B$ and $J$ are real two-forms and $\Omega$ is a complex form of degree $k$. $k$ is the type of $\Phi$ or of the generalised almost complex structure. Then a type 0 spinor is a $B$-transform of $e^{i J}$ which in turn has non-zero norm if $J$ is non-degenerate and is closed if $d J=0$. Hence it corresponds to a generalised complex structure that is derived from a symplectic structure as in (2.3). Similarly a type 3 spinor corresponds to a generalised almost complex structure derived from a complex structure as in (2.2). Then a general pure spinor is some combination of the two. We note here that in general the type of the spinor may vary throughout the manifold jumping by even numbers at particular loci on the manifold. It can also be seen that a $B$-transform preserves the type of the spinor while a $\beta$-transform in general need not.

\section{An example: $\mathbb{C}_{2}$}

In order to illustrate the notions defined above we consider the simple model of $\mathbb{C}_{2}$

In this section we outline a very simple example of how the constructions described in the previous sections can be realised on the manifold $\mathbb{C}_{2}$. Consider the standard complex structure on $\mathbb{C}_{2}$ defined by the spinor

$$
\Phi=d z_{1} \wedge d z_{2}
$$

In terms of the matrix notation the generalised complex structure reads

$$
\mathcal{J}=\left(\begin{array}{cccc}
0 & 1 & 0 & 0 \\
-1 & 0 & 0 & 0 \\
0 & 0 & 0 & 1 \\
0 & 0 & -1 & 0
\end{array}\right)
$$

Now we may perform a $\beta$ transform. Since we have a full generalised complex structure rather than a generalised almost complex structure we should take the bivector to be holomorphic $\beta=z_{1} \partial_{z_{1}} \wedge \partial_{z_{2}}$. Then performing a transform we find a new spinor

$$
\Phi_{\beta}=z_{1}+d z_{1} \wedge d z_{2}
$$

This is still a generalised complex structure, but now it is not simply induced by a complex structure. Rather it exhibits the jumping phenomenon between a generalised complex structure induced by a complex structure and one induced by a symplectic structure. To see this note that at the locus $z_{1}=0$ we recover the original type 2 generalised complex structure. Away from this locus we can write the spinor as

$$
\Phi_{\beta}=z_{1} e^{\frac{d z_{1} \wedge d z_{2}}{z_{1}}}=z_{1} e^{B+i J},
$$

which is a ( $B$-transform of a) generalised complex structure of type 0 , induced by a symplectic structure on the manifold.

\subsection{Generalised geometry and non-geometry}

In this section we outline the connection between generalised geometry as described in the previous sections and the physical view of non-geometry. The motivation is to reconcile the work in [8, 39] and [51] with the discussion of the previous sections. 
We approach non-geometry by considering a $T^{2}$ fibration over an $S^{1}$ given by the metric,

$$
d s^{2}=d x^{2}+d y^{2}+d z^{2}
$$

where $x$ is the co-ordinate along the circle and $y, z$ are the co-ordinates on the $T^{2}$. As we go around the $S^{1}$ we can identify the torus co-ordinates up to a $S L(2, \mathbb{Z})$ transformation which is the symmetry group of the torus. Now we put $m$ units of $H$ flux through the manifold with a $B$-field given by

$$
B=m x d y \wedge d z
$$

It was shown in [7, 8] that if we perform a T-duality along one of torus direction we reach a twisted-torus where now the manifold has torsion but there is no NS flux. The NS flux has been exchanged for metric fluxes. Performing another T-duality we reach the configuration

$$
\begin{aligned}
d s^{2} & =\frac{1}{1+m^{2} x^{2}}\left(d y^{2}+d z^{2}\right)+d x^{2} \\
B & =\frac{m x}{1+m^{2} x^{2}} d y \wedge d z
\end{aligned}
$$

We now see that if we go around the $S^{1}$ the $T^{2}$ metric is not periodic under an $S L(2, \mathbb{Z})$ transformation but rather under $O(2,2, \mathbb{Z})$. This new symmetry group corresponds to the geometric $S L(2, \mathbb{Z})$ plus T-dualities. Therefore this background can not be described geometrically in the usual sense. It is termed non-geometric or a T-fold.

In [39] a way of dealing with non-geometric manifolds was proposed. The basic idea is to double the number of dimensions so that $T^{2} \rightarrow T^{4}$, or more generally $T^{n} \rightarrow T^{2 n}$ for an $n$ torus. Then the geometric symmetry group becomes $S L(2 n, \mathbb{Z})$. The extra dimensions, or degrees of freedom, are then eliminated by a constraint which breaks the symmetry group down to $O(n, n, \mathbb{Z})$. Now this group is large enough to accommodate the T-dualities as well. The way this is realised in string theory is as follows. For a compactification on $T^{n}$, the internal momenta $P^{i}, i=1, . ., n$, combine with the winding modes $w^{i}$ to form the momenta $P_{L}^{i}=P^{i}+w^{i}$ and $P_{R}^{i}=P^{i}-w^{i}$. Then the conjugate coordinates are written as $X_{L}^{i}=X^{i}+\tilde{X}^{i}$ and $X_{R}^{i}=X^{i}-\tilde{X}^{i}$, and $X^{i}$ are T-dual to $\tilde{X}^{i}$. The doubled formalism treats this as a compactification on $T^{2 n}$ with coordinates $X$ and $\tilde{X}$, and the constraint that halves the degrees of freedom is imposing that $X_{L}$ and $X_{R}$ are left-moving and right-moving coordinates. Practically, this can be done by considering a projection of the doubled torus to a product torus $T^{2 n} \rightarrow T^{n} \oplus \tilde{T}^{n}$ such that the torus $T^{n}$ is identified with space-time and $X$ are the space-time coordinates. The CFT on the world sheet is invariant under which projection, or polarisation, we choose to make, i.e. we can rotate the fields $X$ and $\tilde{X}$ into each other. Then T-duality can be viewed as a rotation on the projectors. The failure to patch a manifold with geometric transition functions then corresponds to a failure to have a global polarisation.

The connection to generalised geometry is that under the space-time projection $T^{2 n} \rightarrow T^{n}$ the tangent bundle of the double torus is projected to the sum of the cotangent and tangent bundles of the space-time torus $T\left(T^{2 n}\right) \rightarrow\left(T \oplus T^{*}\right)\left(T^{n}\right)$. Then choosing the space-time $T^{n}$ within the $T^{2 n}$ is equivalent to choosing a maximal isotropic subspace or a pure spinor, which under the projection of the tangent bundle leads to a pure spinor, or a maximal isotropic subspace, or a generalised almost complex structure in $T \oplus T^{*}$. Then T-duality, which is a rotation of the projectors, is given by a rotation of $\mathcal{J}$. More precisely the full symmetry group 
$O(n, n, \mathbb{Z})$ is simply the symmetry group of the generalised metric, elements of which can be split into three types $\{\beta, \varphi, B\}$ where $\varphi$ are elements of the geometric $S L(n, \mathbb{Z})$ subgroup. Then $B$ and $\beta$-transforms are non-geometric symmetries. The $B$-transforms are identified with shifts of the $B$-field that mix the metric and $B$-fields, and the $\beta$-transforms are identified with Tdualities. Patching a manifold with T-dualities then corresponds to patching with $\beta$-transforms. This way we have a (generalised) geometric formulation of non-geometry.

There is another side to non-geometry that we have not mention so far. This is the issue of non-commutativity. It was shown in 64 that performing two T-dualities with flux leads to a non-commutative space. This was related to generalised geometry in [64, 51] where it was shown that the non-commutativity is felt by open strings that end on separate patches which are patched by T-dualities. We note then that this non-commutativity only affects the open string sector and therefore does not alter the analysis in this paper. It is worth mentioning that there are other non-geometric fluxes, denoted by $R$ in the literature [41, that arise from three T-dualities along directions with H-flux . These lead to non-associative manifolds which are non-geometric even locally.

\subsection{A supergravity analysis}

In this paper we perform a classical supergravity analysis of non-geometric compactifications. Given that non-geometric T-folds are inherently stringy in nature, a supergravity analysis requires some justification. In this section we present some reasoning in support of our approach and argue that our analysis may capture true vacua of the full string theory. We follow in part the discussions presented in [39, 31].

We begin this section with a brief discussion based on mirror symmetry which justifies the supergravity approach. This simply follows from the fact that the mirror duals of non-geometric compactifications are geometric. They can be as simple as a Calabi-Yau compactification with some H-flux. These compactifications are under control from a supergravity perspective and so we would expect that under the mirror identification their duals would also be valid2. The rest of this section is, in a sense, dedicated to suggesting why the mirror symmetry works. That is to explaining why, by using generalised geometry, we can recover the IIA (non-geometric) mirrors of magnetic NS fluxes through a supergravity compactification.

The first problem that a supergravity analysis of a T-fold faces is that the T-dualities that patch the T-fold mix momentum and winding modes. The ten-dimensional supergravity is derived by integrating out all the winding modes and keeping the momentum modes. On any particular patch this is valid, in the language of the doubled formalism we are choosing a polarisation. Globally, such a truncation is not valid since on different patches winding modes may become lighter than momentum modes following a T-duality. However, consider compactifying the ten-dimensional supergravity on a T-fold. To derive a four-dimensional effective theory we must now also integrate out the momentum modes, keeping only the zero mode (which has no winding equivalent). Therefore, given such a truncation of both the winding and momentum modes, any mixing should not affect the four-dimensional theory. In terms of

\footnotetext{
${ }^{2}$ In this paper we require non-geometric fluxes to find Minkowski vacua on both the IIA and IIB sides of the mirror. However, for each case the non-geometric fluxes are dual to geometric fluxes. The IIA non-geometric fluxes are dual to magnetic NS flux, and the IIB non-geometric fluxes are dual to metric fluxes. Therefore we expect that the mirror symmetry reasoning presented above still holds.
} 
this reasoning the energy scale of the low energy theory need not be any lower than in a geometric compactification since, if we are below the scale of a KK mode on a radius $R$, we are also below the scale of a winding mode on a radius $1 / R$.

It is possible that it is the symmetry of the effective four-dimensional theory under Tdualities of the internal manifold that means the four-dimensional supergravity is the mirror to a supergravity derived from a geometric compactification. To see how this symmetry manifests itself in terms of the four-dimensional superpotential consider compactifying on an $S U(3) \times$ $S U(3)$ structure manifold that is patched with $B$ and $\beta$-transforms. As outlined in section 2.3 , this is a compactification on a T-fold. We have two pure spinors $\Phi^{ \pm}$on the manifold, and these would $B$ and $\beta$-transform between different patches. Let us turn off any RR-fluxes for simplicity. We decompose the ten-dimensional NS two-form $\hat{B}$ into a part that leads to the four-dimensional fields (axions) $B$ and a part responsible for background flux $B^{b g}$ so that the background flux $H^{b g}$ is given by $H^{b g}=d B^{b g} 3$. Then the four-dimensional superpotential was derived in 30] and reads

$$
W=\int_{M_{6}}\left\langle\left(d-H^{b g}\right) \tilde{\Phi}^{+}, \tilde{\Phi}^{-}\right\rangle
$$

where we absorbed the axions into the spinors $e^{-B} \Phi^{ \pm}=\tilde{\Phi}^{ \pm}$. The brackets $\langle$,$\rangle denote the$ Mukai pairings and are defined in section 3.1. We can rewrite (2.18) in a suggestive way

$$
W=\int_{M_{6}}\left\langle d\left(e^{-B^{b g}} \tilde{\Phi}^{+}\right),\left(e^{-B^{b g}} \tilde{\Phi}^{-}\right)\right\rangle .
$$

Written this way (2.19) is a $B$-transform of (2.18) with $-B^{b g}$ where we also transform the derivative

$$
d-H^{b g} \rightarrow d-\left(H^{b g}+d\left(-B^{b g}\right)\right)=d .
$$

Viewed in this way we can think of $B$-transforms as gauge transformations with a covariant derivative $\mathcal{D}_{H}=d-H$ that, under a $B$-transform transforms as $D_{H} \rightarrow D_{H}-d B$. The superpotential

$$
W=\int_{M_{6}}\left\langle\mathcal{D}_{H^{b g}} \tilde{\Phi}^{+}, \tilde{\Phi}^{-}\right\rangle
$$

is then invariant under $B$-transforms and so can be integrated over a manifold patched with $B$-transforms to give the same four-dimensional theory.

Now we can also 'gauge' $\beta$-transforms in the same way. The resulting covariant derivative is then

$$
\mathcal{D}_{H, Q}=d-H-Q,
$$

where we have introduced a new 'flux' which is a tensor that under a $\beta$-transform transforms as

$$
Q \rightarrow Q+d \wedge \beta
$$

Indeed the transformation of $Q$ corresponds to the change of the derivative operator under $\beta$-transforms as shown in [51, and can be thought of as encoding this change. It was also

\footnotetext{
${ }^{3}$ This decomposition becomes more subtle in the presence of metric fluxes and is discussed in section 3.3 .1
} 
proposed in 31] by considering the possible fluxes that can be turned on. We now have a covariant derivative of both $B$ and $\beta$-transforms

$$
\mathcal{D}_{H, Q} \Phi^{ \pm} \rightarrow e^{\beta+B}\left(\mathcal{D}_{H, Q} \Phi^{ \pm}\right)
$$

so that the superpotential

$$
W=\int_{M_{6}}\left\langle\mathcal{D}_{H^{b g}, Q^{b g}} \tilde{\Phi}^{+}, \tilde{\Phi}^{-}\right\rangle
$$

is invariant under both $B$ and $\beta$-transforms and so can be integrated over the internal manifold to give the four-dimensional theory. The tensor $Q$ can be thought of as a flux in analogy with $H$ being the NS flux, and in the literature this would be identified with non-geometric flux. In general the operator $\mathcal{D}$ (we henceforth drop the indices) can send the degree of the form it acts on up one or three and if there is also $Q$-flux, down one. Finally we require the condition on the fluxes

$$
i_{Q} H=0
$$

for the operator $\mathcal{D}$ to be nilpotent.

We have seen that, using the covariant derivative $\mathcal{D}$, the ten-dimensional expression for the four-dimensional superpotential is invariant under $B$ and $\beta$-transforms. To derive the effective four-dimensional superpotential in terms of the four-dimensional superfields we should decompose the spinors in terms of a basis of 'low energy' forms. These would be the harmonic forms on a Calabi-Yau but more generally correspond to a truncation to a finite subset of forms [14]. These forms would transform under $\mathrm{B}$ and $\beta$-transforms in the same way as the spinors. However, as we have shown, such a transformation does not change the expression for the superpotential which can still be written in terms of the old basis forms. Therefore the four-dimensional superpotential in terms of the superfields takes the same form on all the patches of the internal T-fold 5

Having discussed the effects of the mixing of the winding and momentum modes let us briefly consider quantum effects. The mirror symmetry argument presented at the beginning of this section should still hold since mirror symmetry should be a quantum symmetry. In the case of non-perturbative effects such as instantons, gaugino condensation, light modes, the size of the effects is determined by integrating the calibration form on the submanifolds they wrap. But these calibration forms are the pure spinors of the generalised geometry [60. Therefore the size of these effects should be measured by the size of the four-dimensional superfields on each patch. Note that, since these appear perturbatively in the superpotential, in vacua where they take large values, non-perturbative corrections to the superpotential will be highly suppressed.

Another possible correction source are $\alpha^{\prime}$ corrections due to the small curvature scales of the T-fold. These could come in the form of corrections to the Kähler potential and corrections to the superpotential. The corrections to the Kähler potential do not affect the supersymmetric Minkowski vacua we are studying in this paper. Corrections to the superpotential may be possible, but in the regime of large fields, which we consider here, such corrections will still

\footnotetext{
${ }^{4}$ Note that also the Kähler potential is invariant since it is given by the Hitchin functional [58, 14] $K=$ $-i \ln \int_{M_{6}}\left\langle\Phi^{ \pm}, \Phi^{ \pm}\right\rangle$.

${ }^{5}$ Note that, as proposed in 31, we could have equally used a normal exterior derivative instead of $\mathcal{D}$ and considered the basis forms to transform so that they become twisted $\omega \rightarrow\left(e^{\beta} \omega\right)$.
} 
be subdominant. Given this reasoning we proceed with the compactifications neglecting any possible $\alpha^{\prime}$ corrections.

To summarise, we have argued that it may be possible to treat the compactifications to Minkowski vacua we are studying in this paper as a four-dimensional supergravity. To remain in the perturbative regime we require the fields to take large values, for the dilaton this implies weak string coupling, for the geometric moduli the interpretation is a little less clear but the limit is required to suppress non-perturbative corrections. We also require to stay below the KK (or equally winding) scale. This is non-trivial to show in the presence of metric fluxes since the basis forms are no longer harmonic. However, we expect that there is such a low energy truncation since one exists in a Calabi-Yau mirror to a manifold with metric fluxes, see also [65, 14, 31] for constraints on these truncations. Therefore, assuming such a possible low energy truncation, we cautiously proceed with the supergravity analysis.

\section{The compactifications}

In this section we derive the superpotential and tadpole constraints of the effective $\mathcal{N}=1$ theory resulting from compactifications of type II theories on orientifolds with $S U(3) \times S U(3)$ structure. In both setups we consider an unwarped compactification given by the product Ansatz of the form $M_{10}=S_{4} \times Y_{6}$. Here $S_{4}$ is an unwarped four dimensional space, while $Y_{6}$ is a six dimensional compact manifold. After deriving the two four-dimensional theories independently we give the mirror map under which they can be identified.

Throughout this paper we work in string units as in 55] where we take the basis forms $\alpha, \beta, \ldots$ to belong to an integer basis so that in units of $2 \pi / \mu_{p-2} l^{p}=1 / l$, where $p$ is the degree of the form, $\mu_{p-2}$ is the Dp-brane unit of charge and $l=2 \pi \sqrt{\alpha^{\prime}}=1$ is the string length, the fluxes are integers.

\subsection{Type IIA compactifications on $S U(3) \times S U(3)$ orientifolds}

The compactification of type IIA string theory on generalised orientifolds was studied in [30] and we follow their results in parts of this section. We begin by specifying the two compatible pure spinors $\Pi^{o d}$ and $\Pi^{e v}$

$$
\begin{aligned}
\Pi^{o d} & =e^{-B} C \Omega, \\
\Pi^{e v} & =e^{J_{c}} \\
J_{c} & \equiv-B+i J,
\end{aligned}
$$

Here $B$ denotes the (internal part of the) NS two-form that leads to the axions. It does not include the background contribution $B^{b g} . \quad J$ is the Kähler form on the manifold, or more generally, for the non-Kähler cases we study, the almost symplectic two-form. We then see that $\Pi^{e v}$ is just a $B$-transform of the generalised almost complex structure induced by $J$. In general $\Pi^{\text {od }}$ can have type one, three or five and may jump between them. For the type three case $J$ and $\Omega$ are the $S U(3)$-structure forms that appear in standard $S U(3)$-structure compactifications (including Calabi-Yau compactifications). $C$ is the compensator field defined as

$$
C \equiv e^{-\hat{\phi}-i \theta} e^{\frac{1}{2}\left(K^{c s}-K^{K}\right)}, e^{-K^{c s}}=i \Omega \wedge \bar{\Omega}, e^{-K^{K}}=\frac{4}{3} J \wedge J \wedge J
$$


where $\hat{\phi}$ is the ten-dimensional dilaton related to the four-dimensional dilaton $\phi$ through

$$
e^{-2 \phi}=e^{-2 \hat{\phi}} \frac{4}{3} \int_{Y} J \wedge J \wedge J
$$

The angle $\theta$ is fixed by the calibration condition for the orientifolds. In this paper we are interested in the compactifications that are mirror to type IIB compactifications with $03 / O 7$ orientifolds in which case we choose the orientation of the $O 6$ planes so that $\theta=0$. The relevant ten-dimensional fields are completed by the RR fields which we define in formal sums

$$
C^{o d} \equiv C_{1}+C_{3}+C_{5}+C_{7}+C_{9} .
$$

Note that only half the degrees of freedom in (3.6) are physical. We also have the relevant field-strengths

$$
F^{e v}=F_{0}+F_{2}+F_{4}+F_{6},
$$

where $F^{e v}=d_{H} C^{o d}$. It is convenient to define the complex combination

$$
\Pi_{c}^{o d} \equiv \tilde{C}_{(0)}^{o d}+i \operatorname{Re} \Pi^{o d},
$$

where $\tilde{C}^{o d}=e^{-B} C^{o d}$, and the (0) subscript denotes the component that is a four-dimensional scalar. The four-dimensional superpotential resulting from this compactification was derived in [30] by reduction of the gravitino mass term. Here we use their expression but replace the twisted derivative with the full covariant derivative $d_{H^{b g}} \rightarrow \mathcal{D}$ as explained in the previous section. With the definitions above the superpotential reads

$$
W^{I I A}=\int_{Y}\left\langle\hat{F}^{e v}+\mathcal{D} \Pi_{c}^{o d}, \Pi^{e v}\right\rangle .
$$

The brackets $\langle\ldots, \ldots\rangle$ denote the Mukai pairing of forms defined as

$$
\left\langle\Psi^{1}, \Psi^{2}\right\rangle \equiv \Psi_{(0)}^{1} \wedge \Psi_{(6)}^{2}-\Psi_{(2)}^{1} \wedge \Psi_{(4)}^{2}+\Psi_{(4)}^{1} \wedge \Psi_{(2)}^{2}-\Psi_{(6)}^{1} \wedge \Psi_{(0)}^{2}
$$

for a sums of even forms, and

$$
\left\langle\Psi^{1}, \Psi^{2}\right\rangle \equiv-\Psi_{(1)}^{1} \wedge \Psi_{(5)}^{2}+\Psi_{(3)}^{1} \wedge \Psi_{(3)}^{2}-\Psi_{(5)}^{1} \wedge \Psi_{(1)}^{2},
$$

for sums of odd forms. The subscripts denote the degree of the component of the forms. To derive the four-dimensional spectrum of fields we need to specify a finite set of basis forms on the manifold. Following [14, 30] we consider a finite symplectic form basis

$$
\begin{aligned}
\omega_{\hat{A}} & =\left(1, \omega_{A}\right), \quad \tilde{\omega}^{\hat{A}}=\left(\tilde{\omega}^{A},-\epsilon\right), \\
\alpha_{\hat{K}} & =\left(\alpha_{0}, \alpha_{K}\right), \quad \beta^{\hat{K}}=\left(\beta_{0}, \beta^{K}\right) .
\end{aligned}
$$

The forms have non-vanishing Mukai pairings

$$
\begin{aligned}
\int_{Y}\left\langle\tilde{\omega}^{\hat{A}}, \omega_{\hat{B}}\right\rangle & =\delta_{\hat{B}}^{\hat{A}}, \\
\int_{Y}\left\langle\alpha_{\hat{L}}, \beta^{\hat{K}}\right\rangle & =\delta_{\hat{L}}^{\hat{K}} .
\end{aligned}
$$


In general the forms $\alpha_{\hat{K}}$ and $\beta^{\hat{K}}$ can have components that are one, three and five-forms. However, since the expression for the superpotential does not depend on which patch, or 'gauge', we are on, we can always consider a local $S U(3)$ structure where $\alpha_{\hat{K}}$ and $\beta^{\hat{K}}$ are three-forms. Under the orientifold projection the forms decompose into [30]

$$
\begin{aligned}
\left(1, \omega_{a}, \tilde{\omega}^{a}, \epsilon\right) & \in \Delta_{+}^{e v}, \\
\left(\omega_{\alpha}, \tilde{\omega}^{\alpha}\right) & \in \Delta_{-}^{e v}, \\
\left(\alpha_{0}, \alpha_{\lambda}, \beta^{k}\right) & \in \Delta_{+}^{o d}, \\
\left(\alpha_{k}, \beta^{0}, \beta^{\lambda}\right) & \in \Delta_{-}^{o d} .
\end{aligned}
$$

The sets $\Delta_{ \pm}$denote forms that are even or odd under the orientifold projection. The index ranges are therefore $a=1, \ldots, \operatorname{dim} \Delta_{+}^{2}, \alpha=1, \ldots, \operatorname{dim} \Delta_{-}^{2}$. For the three-forms we consider the index ranges $k=1, \ldots, \operatorname{dim} \Delta_{+}^{o d}-1$ and $\lambda=\{\varnothing\}$. This is a choice we have in the definition of the basis forms 6 . Under the orientifold splitting we have that

$$
\begin{aligned}
& \Pi^{e v} \in \Lambda_{+}^{e v}, \\
& \Pi_{c}^{o d} \in \Lambda_{+}^{o d} .
\end{aligned}
$$

Therefore we decompose the forms into four-dimensional superfields as

$$
\begin{aligned}
& \Pi^{e v}=1+i T^{a} \omega_{a}-\frac{1}{2} T^{a} T^{b} K_{a b c} \tilde{\omega}^{c}-\frac{i}{6} T^{a} T^{b} T^{c} K_{a b c} \epsilon, \\
& \Pi_{c}^{o d}=i S \alpha_{0}+i U_{k} \beta^{k},
\end{aligned}
$$

where we have introduced the analog of the intersection numbers

$$
K_{a b c} \equiv \int_{Y} \omega_{a} \wedge \omega_{b} \wedge \omega_{c}
$$

We write the superfields in terms of real component fields as

$$
\begin{aligned}
T^{a} & =t^{a}+i b^{a}, \\
U_{k} & =u_{k}+i \nu_{k}, \\
S & =s+i \sigma .
\end{aligned}
$$

The imaginary components of the superfields are usually referred to as axions, a terminology that we keep, even though they all lose their axionic shift symmetries once fluxes are turned on. We refer to the fields $t^{a}$ as the Kähler moduli and to $u_{k}$ as the complex structure moduli, again keeping in mind that these manifolds need not be Kähler or complex. Finally the field $s$ is the related to the dilaton through the definition of the compensator field $C$.

The fluxes transform under the orientifold action such that

$$
\begin{aligned}
F^{e v} & \in \Lambda_{+}^{e v}, \\
H & \in \Lambda_{-}^{3} .
\end{aligned}
$$

\footnotetext{
${ }^{6}$ The more general case where we allow a non-trivial index range for $\lambda$ does not change anything in a fundamental way, rather we just need to turn on appropriate fluxes to recover the same superpotential. It makes the analysis more complicated however.
} 
Turning on background fluxes raises a delicate issue that is discussed in more detail in section 3.3.1 where the distinction between a background flux and an axion vacuum expectation value is made more rigorous. For now we decompose the field-strengths $H$ and $F^{e v}$ as

$$
\begin{aligned}
F^{e v} & =F^{b g}+d C^{o d}, \\
H & =H^{b g}+d B,
\end{aligned}
$$

and we set

$$
\begin{aligned}
F^{b g} & =-m^{0}+p^{a} \omega_{a}-q_{a} \tilde{\omega}^{a}-e_{0} \epsilon \\
H^{b g} & =-h_{0} \beta^{0}+h^{k} \alpha_{k} .
\end{aligned}
$$

We take the background flux parameters to be integer quantised (ignoring any possible subtleties with half-integer values induced by orientifolds).

To calculate the superpotential we should also specify the differential properties of the basis forms. In general they need not be harmonic, i.e. closed and co-closed. Indeed if there is torsion on the manifold they will not be. The torsion is usually referred to as metric fluxes, an appropriate term when it comes to looking at mirror symmetry where these fluxes are dual to electric NS flux. On our manifold we generally also have non-geometric $Q$-flux, this appears like metric fluxes but corresponds to the degree of the form decreasing by one. They are mirror dual to magnetic NS flux [14, 30, 31]. For the differential relations of the basis forms we take 7

$$
\begin{aligned}
& \mathcal{D} \alpha_{0}=-m^{a} \omega_{a}-e_{a} \tilde{\omega}^{a}-h_{0} \epsilon, \\
& \mathcal{D} \beta^{k}=-e_{a}{ }^{k} \tilde{\omega}^{a}-h^{k} \epsilon, \\
& \mathcal{D} \omega_{a}=-e_{a} \beta^{0}+e_{a}{ }^{k} \alpha_{k}, \\
& \mathcal{D} \tilde{\omega}^{a}=m^{a} \beta^{0} .
\end{aligned}
$$

All other forms are closed. The fluxes $e_{a}$ and $e_{a}{ }^{k}$ are the metric fluxes and $m^{a}$ are the nongeometric fluxes. These relation are not the most general relations, we have not turned on all the possible fluxes. Rather we have turned on the minimum amount of fluxes needed to find the Minkowski vacua. We note that we are in a sense allowing ourselves too much freedom. The metric, and non-geometric, fluxes will be fixed by the particular manifold we are choosing to compactify on. However, all our fluxes, metric and non-geometric, have mirrors that are simply NS fluxes [7, 30, 66, 31] and so should in principle be tunable even though we may not have an explicit manifold for every choice.

Substituting all this into the superpotential (3.9), and integrating over the internal manifold, we find

$$
\begin{aligned}
W^{I I A} & =\frac{i}{6} m^{0} K_{a b c} T^{a} T^{b} T^{c}+\frac{1}{2} K_{a b c} p^{c} T^{a} T^{b}-i q_{a} T^{a}+e_{0} \\
& -\frac{i}{2} S K_{a b c} m^{a} T^{b} T^{c}+S e_{a} T^{a}+e_{a}{ }^{k} U_{k} T^{a}+i h_{0} S+i h^{k} U_{k} .
\end{aligned}
$$

This type of superpotential was already proposed in [49, 55] through mirror symmetry.

\footnotetext{
${ }^{7}$ We have included only the background contribution, $H^{b g}$, of the NS flux to the operator $\mathcal{D}$. This is consistent with our approach in section 3.3.1
} 


\subsubsection{IIA tadpoles and Bianchi identities}

In this section we write the constraints that arise from tadpole constraints/Bianchi identities. In the RR sector we have the tadpole constraint/Bianchi identity 63.

$$
d_{H} F^{e v}=\delta_{\text {source }} .
$$

Again, we generalise this to

$$
\mathcal{D} F^{e v}=\delta_{\text {source }} .
$$

The localised RR charged sources are denoted by $\delta_{\text {source }}$ and can be wrapped $D 6$ branes or $O 6$ orientifolds. It is important to notice that the full field-strengths, rather than just the background ones, appear in the relations. Hence, in general, there is a contribution from the vacuum expectation values of the axions. This issue is discussed in more detail in section 3.3 .1 and for now we only include the background flux $H^{b g}$ in the expressions. Putting the expressions for the field-strengths into the Bianchi identity we find

$$
\left(-m^{0} h_{0}-p^{a} e_{a}-m^{a} q_{a}\right) \beta^{0}-\left(m^{0} h^{k}+p^{a} e_{a}{ }^{k}\right) \alpha_{k}=\delta_{\text {source }} .
$$

Following [15, 63] we proceed to 'smear' the localised sources so that (3.32) can be solved for each component. This involves replacing the localised sources with integer charges multiplying an appropriate form. The sign of the resolved forms is fixed by an appropriate calibration condition as in 63]. Where for a resolved form $\gamma$ we require 8

$$
\left\langle\operatorname{Im}\left(\Pi_{c}^{o d}\right), \gamma\right\rangle \sim+\epsilon .
$$

With the sign fixed in this way we reach the constraints

$$
\begin{aligned}
-m^{0} h_{0}-p^{a} e_{a}-m^{a} q_{a} & =Q_{0} \equiv 2 N_{0}^{D 6}-4 N_{0}^{O 6}, \\
-m^{0} h^{k}-p^{a} e_{a}^{k} & =Q^{k} \equiv 2 N^{k, D 6}-4 N^{k, O 6},
\end{aligned}
$$

where $N^{k, 06 / D 6}$ denotes the number of $O 6 / D 6$ planes wrapped on the submanifold $\beta^{k}$. The Bianchi identity for the NS flux reads $d H=0$. Note that using the condition for $\mathcal{D}$ to be nilpotent, (2.26), the Bianchi identity can be written as $\mathcal{D} H=0$ which is identically satisfied for our choice of fluxes. Finally, the differential relations for the basis forms (3.28) impose a self consistency constraint, obtained by taking two derivatives

$$
m^{a} e_{a}^{k}=0
$$

Notice that we do not consider any non-Abelian gaugings to be generated by ((non)-geometric) fluxes and therefore the number of constraints which we have for the parameters are less than in the twisted tori case 9 .

\footnotetext{
${ }^{8}$ There is a minus sign with respect to the conventions of 63 due to the different definitions of the Mukai pairings.

${ }^{9}$ We thank Pablo Camara for pointing us this fact.
} 


\subsection{Type IIB compactifications on $S U(3) \times S U(3)$ orientifolds}

These compactifications follow in a very similar way to the IIA compactifications discussed in section 3.1 and so we just outline the important steps. The orientifolds we consider are of the O3/O7 type. The compactification was also studied in [30].

The pure spinors are

$$
\begin{aligned}
\Phi^{o d} & \equiv e^{-B} \Omega, \\
\Phi^{e v} & \equiv e^{-\hat{\phi}} e^{J_{c}} .
\end{aligned}
$$

The RR form fields are all even and we define the formal sums

$$
\begin{aligned}
& C^{e v} \equiv C_{0}+C_{2}+C_{4}+C_{6}+C_{8} \\
& F^{o d} \equiv F_{1}+F_{3}+F_{5} .
\end{aligned}
$$

We form the complex superfield combination

$$
\Phi_{c}^{e v} \equiv C_{(0)}^{e v}+i \operatorname{Re} \Phi^{e v}
$$

where $C_{(0)}^{e v}$ denotes the component of $C^{e v}$ that is a four-dimensional scalar. With this the superpotential reads 30 ]

$$
W^{I I B}=\int_{\tilde{Y}}\left\langle F_{3}+\mathcal{D} \Phi_{c}^{e v}, \Phi^{o d d}\right\rangle,
$$

where $\tilde{Y}$ denotes the internal manifold, and we have replaced $d_{H} \rightarrow \mathcal{D}$. In order to compute the four-dimensional spectrum we restrict to a finite symplectic basis of forms as in (3.16). Under the orientifold projection these split as

$$
\begin{aligned}
\left(1, \omega_{\alpha}, \tilde{\omega}^{a}\right) & \in \Delta_{+}^{e v} \\
\left(\omega_{a}, \tilde{\omega}^{\alpha}, \epsilon\right) & \in \Delta_{-}^{e v} \\
\left(\alpha_{0}, \alpha_{k}, \beta_{0}, \beta^{k}\right) & \in \Delta_{-}^{o d} \\
\left(\alpha_{\lambda}, \beta^{\lambda}\right) & \in \Delta_{+}^{o d}
\end{aligned}
$$

As in the IIA case we truncate the spectrum so that the index $\alpha=\{\varnothing\}$. Under the orientifold action we have that

$$
\begin{aligned}
& \Phi_{c}^{e v} \in \Lambda_{+}^{e v}, \\
& \Phi^{o d} \in \Lambda_{-}^{o d},
\end{aligned}
$$

and so we decompose the spinors into superfields as

$$
\begin{aligned}
\Phi_{c}^{e v} & =i \tau-i T_{a} \tilde{\omega}^{a} \\
\Phi^{o d d} & =\alpha_{0}+i U^{k} \alpha_{k}+\frac{1}{2} U^{k} U^{l} \kappa_{k l m} \beta^{m}-\frac{i}{6} U^{k} U^{l} U^{m} \kappa_{k l m} \beta^{0} .
\end{aligned}
$$


Here the coefficients $\kappa_{k l m}$ feature in the prepotential for the complex structure moduli. We write the superfields in terms of their real component fields as

$$
\begin{aligned}
T_{a} & =t_{a}+i b_{a}, \\
U^{k} & =u^{k}+i \nu^{k}, \\
\tau & =e^{-\hat{\phi}}+i \sigma .
\end{aligned}
$$

We now turn to specifying the fluxes. The background fluxes we consider are

$$
\begin{aligned}
H^{b g} & =m^{k} \alpha_{k}-e_{k} \beta^{k}+h_{0} \beta^{0}, \\
F_{3}^{b g} & =-m^{0} \alpha_{0}+p^{k} \alpha_{k}+q_{k} \beta^{k}-e_{0} \beta^{0} .
\end{aligned}
$$

The metric and non-geometric fluxes are specified in the differential relations for the forms which we take to be

$$
\begin{aligned}
& \mathcal{D} \alpha_{0}=h^{a} \omega_{a}+h_{0} \epsilon, \\
& \mathcal{D} \alpha_{k}=-e_{k}{ }^{a} \omega_{a}-e_{k} \epsilon, \\
& \mathcal{D} \tilde{\omega}^{a}=-e_{k}^{a} \beta^{k}+h^{a} \beta^{0} .
\end{aligned}
$$

With these conventions the superpotential (3.41) evaluates to

$$
\begin{aligned}
W^{I I B} & =\frac{i}{6} m^{0} \kappa_{k l m} U^{k} U^{l} U^{m}+\frac{1}{2} \kappa_{k l m} p^{k} U^{l} U^{m}-i q_{k} U^{k}+e_{0} \\
& -\frac{i}{2} \tau \kappa_{k l m} m^{k} U^{l} U^{m}+T_{a} e_{k}^{a} U^{k}+i h^{a} T_{a}+\tau e_{k} U^{k}+i h_{0} \tau .
\end{aligned}
$$

\subsubsection{IIB tadpoles and Bianchi identities}

The Bianchi identities or tadpole conditions for the IIB case read 63 .

$$
\mathcal{D} F^{o d}=\delta_{\text {source }} .
$$

Putting in the expressions for the field-strengths and regularising the sources we find

$$
\begin{aligned}
-m^{0} h_{0}-m^{k} q_{k}-e_{k} p^{k} & =Q_{0} \equiv 2 N^{D 3}-\frac{1}{2} N^{O 3} \\
-p^{k} e_{k}^{a}-m^{0} h^{a} & =Q^{a} \equiv 2 N^{a, D 7}-8 N^{a, O 7} .
\end{aligned}
$$

We also have the constraint arising from the Bianchi identity for the NS field $\mathcal{D} H=0$

$$
m^{k} e_{k}^{a}=0 \text {. }
$$

\subsection{The mirror map}

The two four-dimensional theories derived in sections 3.1 and 3.2 were constructed as a mirror pair. It is clear that the superpotentials (3.29) and (3.51), and pure spinors (3.18) and (3.47), match under the identification of the superfields

$$
\tau \leftrightarrow S, \quad U^{k} \leftrightarrow T^{a}, \quad U_{k} \leftrightarrow T_{a}
$$


and the basis forms

$$
1 \leftrightarrow \alpha_{0}, \omega_{a} \leftrightarrow \alpha_{k}, \quad \tilde{\omega}^{a} \leftrightarrow-\beta^{k}, \epsilon \leftrightarrow \beta^{0},
$$

The mirror fluxes on each side are denoted by the same symbols. Note that on the IIB side $\tau$ is the ten-dimensional dilaton and on the IIA side $S$ is the compensator field defined in (3.4). The actual equivalence is at the four-dimensional dilaton level. The quantity we should keep large is the inverse string coupling which is the ten-dimensional dilaton

$$
g_{s}^{-1}=e^{-\hat{\phi}} \gg 1
$$

\subsubsection{Exact flux and axions}

In section 3.1 we faced the problem of whether to include the contribution from the axions, $b^{a}$, to the tadpole equations. Recall that due to the non-closure of the forms in which we expand, a non-trivial vacuum expectation value for the $B$-field on the internal manifold can lead to a change in the $H$-flux. The tadpole conditions as stated in (3.35) only include a contribution from a 'background' flux $H^{b g}$. From a ten-dimensional point of view however, the Bianchi identities are not sensitive to the splitting between the background flux and the vacuum expectation values for the axions. This motivates the argument that the axions vevs should feature in the tadpole conditions. With the axion contributions the tadpole conditions (3.35) read

$$
\begin{aligned}
\left(-m^{0} e_{a}<b^{a}>\right)-m^{0} h_{0}-p^{a} e_{a}-m^{a} q_{a} & =Q_{0} \\
\left(-m^{0} e_{a}^{k}<b^{a}>\right)-m^{0} h^{k}-p^{a} e_{a}^{k} & =Q^{k}
\end{aligned}
$$

where $\left\langle b^{a}>\right.$ denoted the vacuum expectation value for the scalar field $b^{a}$. Therefore, in a vacuum with non-vanishing combinations $e_{a} b^{a}$ or $e_{a}{ }^{k} b^{a}$, if we solve the conditions without the axions (3.35) then we do not solve the ten-dimensional Bianchi identities.

Even if the argument above for taking into account the axion vevs into the tadpole conditions sounds convincing, there are also other things one should consider. One of the main problems to be addressed is the fact that on the mirror IIB side this does not seem to have any obvious analog. This problem arises even in the simplest cases of half-flat manifolds as in [7]. A possible resolution could be that we should only identify the two theories in a particular vacuum, and this would be one where the axion combinations $e_{a} b^{a}$ and $e_{a}{ }^{k} b^{a}$ vanish. As the IIB mirrors to the NS axions are the real parts of the complex structure moduli, it may be that (see footnote 23 in 63]) non trivial values for them break isometries of the manifold that are required to perform the mirror symmetry. Another possible way out is if we consider the NS flux on the IIB side to be sourced by NS5-branes wrapped on cycles dual to the ones with flux. The calibration condition for the branes is then the mirror condition to requiring that the axions on the IIA side vanish.

In this work, we adopt the following a pragmatic approach that is correct in both cases and which is at most a superfluous constraint on the fluxes. We look for vacua where the combinations $e_{a} b^{a}$ and $e_{a}{ }^{k} b^{a}$ vanish. We implement this practically by assuming that these combinations vanish and using the tadpole conditions (3.35) to solve for the fields. Then choose the fluxes so that in the vacuum this condition is satisfied thereby justifying our assumption. This procedure is clarified in the next section, where we present explicit solutions. 


\section{Supersymmetric Minkowski vacua}

In this section we analyse the vacuum structure of the superpotentials (3.29) and (3.51). We search for supersymmetric Minkowski vacua that are solutions to the equations

$$
\partial_{T^{a}} W=\partial_{U_{k}} W=\partial_{S} W=W=0 .
$$

We begin this section with some no-go theorems regarding the existence of physical Minkowski vacua in our set-up, placing constraints on the type of manifold we should compactify on. In section 4.2 we focus on cases with a reduced number of fields, and study explicit realisations of Minkowski vacua. Then, in section 4.2.4, we provide some arguments to tackle the general case. Throughout this section we work in type IIA notation, with the superpotential given in formula (3.29).

We note that in [13, 55, 49] searches for Minkowski vacua were conducted and examples of supersymmetric Minkowski vacua were found but with not all of the moduli fixed. Some arguments regarding constraints on fixing all the moduli, similar to the no-go theorems we are going to discuss, were consequently presented.

\subsection{Some no-go theorems}

In this section we note two conditions that the compactification manifold must satisfy for Minkowski vacua with all the moduli stabilised to exist. We work in a completely perturbative regime and all our statements are with respect to the superpotential (3.29). More fluxes, non-perturbative or higher order effects would change the superpotential, and mean that our statements are not applicable. In section 4.1.1 we show that for the IIA superpotentials we are considering, in the absence of non-geometric fluxes there are no Minkowski vacua at finite volume with all the moduli stabilised 10. In section 4.1.2 we show that in IIA the number of Kähler moduli must be larger than the number of complex structure moduli in order for all the moduli to be stabilised, with the mirror statement also holding in IIB.

\subsubsection{No SUSY Minkowski with all moduli stabilised for IIA/O6 without non- geometric fluxes}

We set out to show that without non-geometric fluxes it is impossible to stabilise all the moduli in a supersymmetric Minkowski vacuum. The general superpotential we consider is given by (3.29). In order to end with a Minkowski vacuum, we must impose (4.1). Let us consider the following combination, that must vanish at the minimum we are interested in

$$
\operatorname{Im}\left(t^{a} \partial_{T^{a}} W+u^{k} \partial_{U^{k}} W+s \partial_{S} W-W\right)=0 .
$$

A straightforward computation using the superpotential (3.29) shows that the condition (4.2) can be rewritten as

$$
\frac{m^{0}}{3} K_{a b c} t^{a} t^{b} t^{c}=s K_{a b c} m^{a} t^{b} t^{c} .
$$

If we consider vanishing non-geometric fluxes, $m^{a}=0$ for all $a$, the right hand side of (4.3) vanishes. The left hand side is proportional to the volume of the compactification manifold.

\footnotetext{
${ }^{10}$ The IIB case is much simpler, since the dependence on Kahler moduli only comes from non-geometric fluxes.
} 
This implies that the only way to satisfy this relation is to choose $m^{0}=0$. This leaves us with a superpotential that is at most quadratic in the fields.

To proceed with the argument, it is convenient to combine the superfields into one set of fields $\tilde{T}^{\Sigma} \equiv\left(T^{a}, S, U^{k}\right)$, with index $\Sigma, \Lambda=1, \ldots, \operatorname{dim} \Delta_{+}^{2}+\operatorname{dim} \Delta_{+}^{o d}$. We decompose the fields into real and imaginary part as $\tilde{T}^{\Sigma}=\tilde{t}^{\Sigma}+i \tilde{\tau}^{\Sigma}$. The superpotential (3.29) can consequently be rewritten as

$$
W=b_{\Sigma \Lambda} \tilde{T}^{\Sigma} \tilde{T}^{\Lambda}+i c_{\Sigma} \tilde{T}^{\Sigma}-e_{0}
$$

where $b_{\Sigma \Lambda}$ is a square, symmetric, real matrix and $c_{\Sigma}$ a real vector, both depending only on the flux parameters. The condition for a supersymmetric vacuum in Minkowski space, $\partial_{\tilde{T}^{\Sigma}} W=0$ decomposed into real and imaginary parts reads

$$
\begin{aligned}
b_{\Sigma \Lambda} \tilde{t}^{\Lambda} & =0, \\
2 b_{\Sigma \Lambda} \tilde{\tau}^{\Lambda}+c_{\Sigma} & =0 .
\end{aligned}
$$

Contracting (4.6) with $\tilde{t}^{\Sigma}$ and using (4.5) we obtain

$$
c_{\Sigma} \tilde{t}^{\Sigma}=0
$$

Note that these equations always have a flat direction as rescaling the fields $\tilde{t}^{\Sigma}$ by some number leaves them unchanged. In principle this can be resolved by the additional constraint $W=0$ which has to be satisfied in a Minkowski vacuum. However it is easy to see that the above equations conspire to eliminate any dependence of $W$ on $\tilde{t}$ at the critical point leaving us with the flat direction. Finally, as this direction is a proper geometric modulus it has no chance of being the QCD axion and therefore leaving it unfixed is undesirable.

\subsubsection{No SUSY Minkowski for IIA with all moduli stabilised if $\operatorname{dim} \Delta_{+}^{2} \leq \operatorname{dim} \Delta_{+}^{o d}-1$}

Recall that the number of complex structure moduli in the compactification is given by $\operatorname{dim} \Delta_{+}^{o d}-$ 1 , and the number of Kähler moduli is given by $\operatorname{dim} \Delta_{+}^{2}$. Then the no-go theorem states that me must have more Kähler moduli than complex structure moduli.

Consider the case $\operatorname{dim} \Delta_{+}^{2} \leq \operatorname{dim} \Delta_{+}^{\text {od }}-1$. We allow $m^{a} \neq 0$ which implies that (4.3) can be solved without imposing that all the cubic terms in the superpotential vanish. Consider the equations $\partial_{T^{a}} W=0$, that depend on the complex structure moduli. These impose the following system of equations

$$
e_{a}^{k} u_{k}=f_{a},
$$

where $f^{a}$ are expressions that depend on the value of the Kähler moduli and the dilaton at the minimum. Since the complex structure moduli $u_{k}$ appear at most linearly in the superpotential, the conditions $\partial_{U_{k}} W=0$ do not depend on these fields. Also the vanishing of the imaginary part of the superpotential adds no extra constraints on the $u_{k} \mathrm{~s}$, and the real part of the superpotential depends on the same linear combination $e_{a}{ }^{k} u_{k}$ as the constraint (4.8). This means that only the combination $e_{a}{ }^{k} u_{k}$ of the complex structure moduli is constrained.

Now consider the real part of $\partial_{U_{k}} W=0$. This imposes that the Kähler moduli must satisfy the following system of equations

$$
t^{a} e_{a}^{k}=0 .
$$


This implies that, since we cannot accept values $t^{a}=0$, the matrix $e_{a}{ }^{k}$ can not have maximal rank. Therefore there is always at least one combination of the fields $u_{k}$ that remains unconstrained. Again, since this direction represents the dimension of a certain cycle it can not serve as the QCD axion and thus leaving it unfixed is undesirable.

\subsection{Supersymmetric Minkowski vacua}

In this section we consider compactifications leading to Minkowski vacua. We consider some tractable cases that we can solve explicitly, although in section 4.2.4 we show that the important features of the simpler cases generalise to a larger number of moduli. In section 4.2.1 we study the case with a single Kähler modulus and no complex-structure moduli. From a supergravity point of view this case only really makes sense on the IIA side since the IIB equivalent would have no Kähler moduli. From a string theory point of view however this is fine and Minkowski vacua for such a case were constructed in [5]. These may be mirrors to the examples we study with no complex structure moduli on the IIA side. In section 4.2 .2 we consider the case with a complex structure modulus, which then requires (at least) two Kähler moduli. In section 4.2.3 we study the case with three Kähler moduli and no complex structure moduli, which is the closest we can get to an explicit example. Finally in section 4.2 .4 we make some statements regarding the general case. We mostly work in IIA notation, although it is clear, at least for the cases with complex structure moduli, that these vacua are valid also in the IIB case under the mirror map outlined in section 3.3 .

\subsubsection{One Kähler modulus and no complex structure moduli}

The index ranges for this case are simply $a=1$ and $k=\{\varnothing\}$. The superpotential (3.29) simplifies to

$$
\begin{aligned}
W & =\frac{i}{6} k m^{0}\left(T^{1}\right)^{3}+\frac{1}{2} k p^{1}\left(T^{1}\right)^{2}-i q_{1} T^{1} \\
& -\frac{i}{2} m^{1} k S\left(T^{1}\right)^{2}+e_{1} S T^{1}+i h_{0} S+e_{0},
\end{aligned}
$$

where $k$ is the single intersection number $k=K_{111}$. The tadpole conditions (3.35) reduce to the single condition

$$
e_{1} p^{1}+m^{0} h_{0}+q_{1} m^{1}=-Q_{0} .
$$

As discussed in section 3.3 .1 we do not include a possible axion contribution. We solve this tadpole equation by taking

$$
q_{1}=-\frac{1}{m^{1}}\left(e_{1} p^{1}+m^{0} h_{0}+Q_{0}\right) .
$$

This eliminates $q_{1}$ from further equations and guarantees that the tadpole is solved. However it also places the constraint on the other fluxes that there is a solution to (4.12) with $q_{1}$ integer.

We proceed to solve the equations (4.1) for the superpotential (4.10). Imposing $\partial_{S} W=0$ gives

$$
\begin{aligned}
b^{1} & =-\frac{e_{1}}{m^{1} k}, \\
\left(t^{1}\right)^{2} & =\frac{2 h_{0}}{m^{1} k}-\frac{e_{1}^{2}}{\left(m^{1} k\right)^{2}} .
\end{aligned}
$$


Since we would like vacua with vanishing axion we henceforth set $e_{1}=0$ thereby justifying not including it in the tadpole condition. The condition $\partial_{T^{1}} W=0$ gives

$$
\begin{aligned}
\sigma & =-\frac{p_{1}}{m^{1}}, \\
s & =\frac{1}{k\left(m^{1}\right)^{2} t^{1}}\left(2 m^{0} h_{0}+Q_{0}\right) .
\end{aligned}
$$

We now wish to impose the condition $W=0$. We begin by solving for a vanishing imaginary part and return to the real part later. The combination

$$
\operatorname{Im}\left(t^{1} \partial_{T^{1}} W+s \partial_{S} W-W\right)=0
$$

becomes the constraint

$$
3 m^{1} s=m^{0} t^{1}
$$

which can be solved by taking

$$
h_{0}=-\frac{3 Q_{0}}{4 m^{0}} .
$$

Again we have the constraint on the fluxes that there is a solution for $h_{0}$ integer. Substituting this into (4.16) and (4.14) we recover

$$
\begin{aligned}
s & =\frac{1}{m^{1}} \sqrt{\frac{-Q_{0} m^{0}}{6 k m^{1}}}, \\
t^{1} & =\sqrt{\frac{-3 Q_{0}}{2 m^{0} m^{1} k}} .
\end{aligned}
$$

Now we see that the value of the Kähler modulus is capped by the orientifold charge. Although we can go to arbitrarily weak coupling this decreases the value for $t^{1}$ such that the product $s t^{1}$ is constant. Note also that if $Q_{0}>0$ there are no solutions. We therefore satisfy the no-go theorem of [63] which states that all the Minkowski vacua must have orientifolds present. We also need to check that the real part of the superpotential vanishes. This can always be done by choosing $e_{0}$ appropriately, with the constraint on the fluxes that it should be integer. For this case the solution is

$$
e_{0}=\frac{3 p^{1} Q_{0}}{4 m_{0} m_{1}} .
$$

We now need to check that we can choose the fluxes such that they are all integer. We first note that the flux $p^{1}$ only features in the value of the axion $\sigma$. Then we are free to choose it as we like without changing the values of the geometric moduli. It is clear that it can be chosen such that $q_{1}$ and $e_{0}$, as written in (4.12) and (4.22), are integers. The flux $h_{0}$, as in (4.19), can be made integer by taking $m^{0}$ to be 1 or 3 , since $Q_{0}$ must be a multiple of 4 . We therefore see that we have a fully consistent solution, which for enough orientifolds, can be at large values for the moduli.

We do not perform any analytic vacua counting since there are too many constraints to be satisfied by the fluxes. We note that, although $p^{1}$ only features in the axion value, the number of vacua is not infinite since in counting vacua we should gauge fix the integer axionic shift symmetry of the superpotential

$$
\sigma \rightarrow \sigma+1, \quad p^{1} \rightarrow p^{1}-m^{1}, \quad q_{1} \rightarrow q_{1}+1, \quad e_{0} \rightarrow e_{0}+h_{0},
$$


by taking

$$
0 \leq p^{1}<\left|m^{1}\right|
$$

There are a number of known nearly-Kähler manifolds that have $S U(3)$-structure which have a single Kähler modulus and no complex-structure moduli, see [67] for a list. We would require a non-geometric deformation of these manifolds to reach the vacua we have found in this section. See section 4.2.3 for a similar point for the case with three Kähler moduli.

To summarise we find Minkowski vacua with all the moduli stabilised and tadpole conditions satisfied. The values of the moduli and dilaton are capped by the orientifolds charge.

\subsubsection{Two Kähler and one complex structure moduli}

We now study the two Kähler moduli and one complex structure modulus case. The index ranges are therefore $a=1,2$ and $k=1$. We have the superpotential

$$
\begin{aligned}
W & =\frac{i}{6} m^{0} k_{0}\left(T^{1}\right)^{3}+\frac{i}{2} m^{0} k_{1} T^{2}\left(T^{1}\right)^{2}+\frac{i}{2} m^{0} k_{2} T^{1}\left(T^{2}\right)^{2}+\frac{i}{6} m^{0} k_{3}\left(T^{2}\right)^{3} \\
& +\frac{1}{2} k_{0} p^{1}\left(T^{1}\right)^{2}+\frac{1}{2} k_{2} p^{1}\left(T^{2}\right)^{2}+\frac{1}{2} k_{1} p^{2}\left(T^{1}\right)^{2}+\frac{1}{2} k_{3} p^{2}\left(T^{2}\right)^{2}+k_{2} p^{2} T^{1} T^{2} \\
& +k_{1} p^{1} T^{1} T^{2}-i q^{1} T^{1}-i q_{2} T^{2}+e_{0} \\
& -\frac{i}{2} k_{2} m^{1} S\left(T^{2}\right)^{2}-\frac{i}{2} k_{1} m^{2} S\left(T^{1}\right)^{2}-\frac{i}{2} k_{3} m^{2} S\left(T^{2}\right)^{2}-i k_{2} m^{2} S T^{1} T^{2}-i k_{1} m^{1} S T^{1} T^{2} \\
& -\frac{i}{2} k_{0} m^{1} S\left(T^{1}\right)^{2}+e_{1} S T^{1}+e_{2} S T^{2}+e_{3} U T^{1}+e_{4} U T^{2}+i h_{0} S+i h^{1} U
\end{aligned}
$$

where we denote $e_{1}{ }^{1}=e_{3}, e_{2}{ }^{1}=e_{4}$. The intersection numbers are denoted as

$$
\begin{aligned}
& k_{0}=K_{111}, \\
& k_{1}=K_{112}, \\
& k_{2}=K_{122}, \\
& k_{3}=K_{222} .
\end{aligned}
$$

The tadpole conditions read

$$
\begin{aligned}
p^{1} e_{1}+p^{2} e_{2}+m^{0} h_{0}+q_{1} m^{1}+q_{2} m^{2} & =-Q_{0}, \\
p^{1} e_{3}+p^{2} e_{4}+m^{0} h^{1} & =-Q^{1}, \\
m^{1} e_{3}+m^{2} e_{4} & =0 .
\end{aligned}
$$

We solve the tadpole conditions by fixing $q_{1}, h_{1}$ and $m_{2}$ in terms of the other fluxes whilst keeping in mind that we have the constraints that they should be integer.

We now go on to solve the supersymmetry variations. The solutions for the axions read

$$
\begin{aligned}
b^{1} & =\frac{m^{0} e_{4}^{2}\left(e_{2} e_{3}-e_{1} e_{4}\right)-\left(e_{4}^{2} k_{1}-2 e_{3} e_{4} k_{2}+e_{3}^{2} k_{3}\right) m^{1}\left(e_{3} p^{1}+e_{4} p^{2}+Q^{1}\right)}{\left(e_{4}^{3} k_{0}-3 e_{3} e_{4}^{2} k_{1}+3 e_{3}^{2} e_{4} k_{2}-e_{3}^{3} k_{3}\right) m^{0} m^{1}}, \\
b^{2} & =\frac{-m^{0} e_{3} e_{4}\left(e_{2} e_{3}-e_{1} e_{4}\right)+\left(e_{4}^{2} k_{0}-2 e_{3} e_{4} k_{1}+e_{3}^{2} k_{2}\right) m^{1}\left(e_{3} p^{1}+e_{4} p^{2}+Q^{1}\right)}{\left(e_{4}^{3} k_{0}-3 e_{3} e_{4}^{2} k_{1}+3 e_{3}^{2} e_{4} k_{2}-e_{3}{ }^{3} k_{3}\right) m^{0} m^{1}} .
\end{aligned}
$$


As discussed in section 3.3 .1 we impose the conditions that the combinations $e_{a} b^{a}$ and $e_{a}{ }^{k} b^{a}$ vanish giving the constraints

$$
\begin{aligned}
e_{2} e_{3} & =e_{1} e_{4}, \\
e_{3} p^{1}+e_{4} p^{2}+Q^{1} & =h^{1}=0 .
\end{aligned}
$$

We can solve these conditions by eliminating $e_{1}$ and $p^{1}$. The moduli values in the vacuum then read

$$
\begin{aligned}
t^{1} & =e_{4} \sqrt{\frac{3}{2}} \sqrt{\frac{-e_{4} Q_{0}+e_{2} Q^{1}}{m^{0} m^{1} F_{1}}}, \\
t^{2} & =-\frac{e_{3}}{e_{4}} t^{1}, \\
s & =\frac{m^{0}}{3 m^{1}} t^{1}, \\
u & =\frac{3 m^{1} Q^{1} F_{2}-e_{2} m^{0} F_{1}}{3 e_{4} m^{1} F_{1}} t^{1},
\end{aligned}
$$

where we define the quantities

$$
\begin{aligned}
& F_{1} \equiv\left(e_{4}\right)^{3} k_{0}-3 e_{3}\left(e_{4}\right)^{2} k_{1}+3 e_{4}\left(e_{3}\right)^{2} k_{2}-\left(e_{3}\right)^{3} k_{3}, \\
& F_{2} \equiv-\left(e_{4}\right)^{2}\left(k_{1}\right)^{2}+\left(e_{4}\right)^{2} k_{0} k_{2}+e_{3} e_{4} k_{1} k_{2}-e_{3} e_{4} k_{0} k_{3}+\left(e_{3}\right)^{2} k_{1} k_{3}-\left(e_{3}\right)^{2}\left(k_{2}\right)^{2} .
\end{aligned}
$$

We have eliminated $h_{0}$ by requiring that the imaginary part of the superpotential vanishes. Its explicit value is given in the appendix along with the values of the axions. Similarly the real part of the superpotential fixes the flux $e_{0}$. The solution (4.31) is a Minkowski vacuum with all the moduli stabilised. The values of the fluxes can be chosen so that all the moduli are positive and large. We later study the number of such vacua, but for now we outline a family of solutions where the moduli can be made parametrically large. Consider setting the fluxes $e_{4}=-e_{3}=m^{0}=m^{1}=1$ and taking $e_{2}$ large. Then the solution for the moduli reads

$$
\begin{aligned}
t^{1}=t^{2}=3 s & \sim \sqrt{\frac{3}{2 F_{1}}} \sqrt{e_{2} Q^{1}}, \\
u & \sim-\frac{1}{3} e_{2} t^{1} .
\end{aligned}
$$

Then if we take $Q^{1}<0$ and $e_{2}<0$ we have a parametrically controlled family of solutions where we can reach arbitrarily large values for the moduli. In terms of the size moduli and string coupling 11 this solution reads

$$
\begin{aligned}
I I A & : \quad t^{1}, t^{2} \sim\left|e_{2}\right|^{\frac{1}{2}}, e^{-\hat{\phi}} \sim\left|e_{2}\right|^{\frac{1}{2}}, \quad u \sim\left|e_{2}\right|^{\frac{3}{2}}, \\
I I B & : \quad u^{1}, u^{2} \sim\left|e_{2}\right|^{\frac{1}{2}}, e^{-\hat{\phi}} \sim\left|e_{2}\right|^{\frac{1}{2}}, \quad t \sim\left|e_{2}\right|^{\frac{3}{2}} .
\end{aligned}
$$

At this point it is worth noting how the parametrically controlled vacua satisfy the no-go theorem of 63. Although it is clear that we require orientifolds wrapped over $\alpha_{1}$, since $Q^{1}<0$,

\footnotetext{
${ }^{11}$ In the type IIA case the ten-dimensional dilaton scales as $e^{-\hat{\phi}} \sim\left(\frac{s u^{3}}{t^{6}}\right)^{\frac{1}{4}}$.
} 
this need not be the case for $Q_{0}$ since it can be positive. At first this leads to an apparent contradiction since we can take $Q_{0}$ large and positive and $Q^{1}$ small and negative (which is fine as long as $e_{2}$ is large and negative) so that if we sum over the total charges present the result is an overall positive charge. The resolution of this is to note that the no-go theorem states that

$$
\int_{Y}\left\langle\operatorname{Im} \Pi_{c}^{\text {od }}, \delta_{\text {source }}\right\rangle=Q_{0} s+Q^{1} u \sim\left|e_{2}\right|^{\frac{1}{2}}\left(Q_{0}+Q^{1}\left|e_{2}\right|\right)<0 .
$$

Hence we see that the charge $Q^{1}$ is weighted by an extra factor of $\left|e_{2}\right|$ thereby satisfying the no-go theorem.

We still have the requirement on the solutions that the tadpoles and other consistency equations are solved for integer values of the fluxes. Because there are may of these constraints it is difficult to perform an analytical estimate of the number of vacua within a flux range. Also since we are not restricted to a particular manifold we have a large number of free parameters. A full analysis of the number of vacua is therefore beyond the scope of this paper. Instead we present a simple analysis, which is intended to give an idea for the numbers. We consider the intersection numbers 12

$$
K_{111}=0, K_{112}=0, K_{221}=4, K_{222}=2 .
$$

We count the number of Minkowski vacua that have all the moduli larger than some value $t^{1}, t^{2}, u, s \geq X_{\min }$ where all the tadpoles vanish and the fluxes take integer values. We also fix the the orientifold/D-branes charges to be $Q_{0}=Q^{1}=-32$. We then scan over the flux parameters $e_{4}, e_{3}, e_{2}, m^{0}, m^{1}$ within an integer range $-M, \ldots, M$. We also have the free fluxes $p^{2}$ and $q_{2}$. However we restrict the values of these fluxes in order to fix left over integer axionic symmetries so as not to overcount the vacua drastically. The symmetries are

$$
\sigma \rightarrow \sigma+1
$$

which can be absorbed into a flux redefinition

$$
e_{0} \rightarrow e_{0}+h_{0}, p^{a} \rightarrow p^{a}-m^{a}, q_{a} \rightarrow q_{a}+e_{a} .
$$

We fix this by constraining

$$
0 \leq p^{2}<\left|m^{2}\right|=-\frac{m^{1} e_{3}}{e_{4}}
$$

Similarly we fix the shifts of $\nu$ by constraining

$$
0 \leq q_{2}<\left|e_{4}\right|
$$

With these constraints the number of vacua are presented in table 1. Note that the vacua are relatively sparse. This is primarily because the constraint on $e_{0}$ being integer which is difficult to satisfy.

To summarise, we find that in the case of two Kähler moduli and one complex structure modulus there is a parametrically controlled family of Minkowski vacua with arbitrary large values for the moduli. In section 4.2.4 we show that this situation is generic as long as we have at least one complex structure modulus.

\footnotetext{
${ }^{12}$ The intersection numbers are those of the complete intersection Calabi-Yau $\left(\begin{array}{lll}2 & 3 \\ 3 & 4\end{array}\right)$.
} 


\begin{tabular}{||c|c|c|c|c|c||}
\hline$M / X_{\min }$ & 1 & 2 & 3 & 4 & 5 \\
\hline 10 & 33 & 0 & 0 & 0 & 0 \\
\hline 20 & 206 & 5 & 0 & 0 & 0 \\
\hline 30 & 481 & 33 & 0 & 0 & 0 \\
\hline 40 & 898 & 94 & 5 & 0 & 0 \\
\hline 50 & 1525 & 250 & 48 & 12 & 6 \\
\hline
\end{tabular}

Table 1: Table showing the number of vacua with all the moduli larger than $X_{\min }$ as a function of the flux parameters range $M$ for the intersection number choices (4.36) and the orientifold charges $Q_{0}=Q_{1}=-32$.

\subsubsection{Three Kähler moduli and no complex structure moduli}

In this section we consider the case of three Kähler moduli and no complex moduli. The main reason for studying this is that $S U(3)$ structure examples of these manifolds are known. For example the tori in [68] and the coset manifold in [12]. We therefore require non-geometric deformations of these manifolds in order to reach the type of superpotentials we are studying. These could be obtained by T-dualising the torus with $\mathrm{H}$-flux along two directions and then modding out by a $Z_{3} \times Z_{3}$ orbifold symmetry. We leave a solid construction of such manifolds for future work and go on to study the superpotential. Since both the known examples are parallelisable manifolds on which the two-forms are constructed as a product of one-forms, the only non-vanishing intersection number is the one with no repeated indices. We make the simplifying assumption that this is also the case at hand and take the only non-vanishing intersection

$$
K_{123}=1
$$

With this the superpotential reads

$$
\begin{aligned}
W= & i m^{0} T^{1} T^{2} T^{3}+p^{1} T^{2} T^{3}+p^{2} T^{1} T^{2}+p^{3} T^{1} T^{2}-i q_{1} T^{1}-i q_{2} T^{2}-i q_{3} T^{3}+e_{0} \\
& -i S m^{1} T^{2} T^{3}-i S m^{2} T^{1} T^{3}-i S m^{3} T^{1} T^{2}+S e_{1} T^{1}+S e_{2} T^{2}+S e_{3} T^{3}+i h_{0} S
\end{aligned}
$$

Since there are no new features in this type of superpotential rather than solving it generally we look for a particular solution. It is easy to find a solution where the fields are all equal and the fluxes with the varying indices are set equal. Then solving the supersymmetry equations is equivalent to solving the one Kähler modulus case as in section 4.2.1. The solution reads

$$
\begin{aligned}
b^{1} & =b^{2}=b^{3}=0, \\
t^{1} & =t^{2}=t^{3}=\sqrt{\frac{-Q_{0}}{4 m^{0} m}}, \\
\sigma & =-\frac{p}{m}, \\
s & =\frac{m^{0}}{3 m} \sqrt{\frac{-Q_{0}}{4 m^{0} m}},
\end{aligned}
$$

where we solved the tadpoles by setting

$$
q=-\frac{1}{3 m}\left(m^{0} h_{0}+Q_{0}\right) .
$$




\subsubsection{The general case}

In this section we discuss the general case where the number of moduli fields is arbitrary up to the constraint $\operatorname{dim} \Delta_{+}^{2}>\operatorname{dim} \Delta_{+}^{o d}-1$. Then equation (4.8) means that if the matrix $e_{a}^{k}$ does not have maximal rank we can not stabilise all the complex structure moduli. We set out to show that if $e_{a}{ }^{k}$ does have maximal rank then there are always solutions with all the moduli stabilised. Further we show that, as long as there is at least one complex structure modulus, these solutions are ones where the moduli can be parametrically taken to arbitrary large values. We do this by outlining a particular class of solutions, rather than finding the most general vacuum. We do not impose the integer constraints on the fluxes and assume that, by performing scans like the one in section 4.2.2, solutions can be found where all the fluxes are integers.

Before proceeding it helps to introduce some notation for the index ranges of the fluxes and moduli. Let there be $n=\operatorname{dim} \Delta_{+}^{2}$ Kähler moduli with the index $a$ running from $1, \ldots, n$. Then we introduce the indices $\tilde{a}$ and $\bar{a}$ which have the ranges $\tilde{a}=1, \ldots, p$ and $\bar{a}=p+1, \ldots, n$, where $p$ is the number of complex structure moduli $p=\operatorname{dim} \Delta_{+}^{o d}-1$.

We now go through the conditions for a supersymmetric Minkowski vacuum and indicate the degrees of freedom fixed by each condition. We look for a solution where all the NS axions vanish $b^{a}=0$. This is sufficient to have no ambiguity in the tadpole conditions.

We start with the condition on the superpotential vanishing. The imaginary part of this condition reads

$$
\frac{m^{0}}{3} K_{a b c} t^{a} t^{b} t^{c}=s K_{a b c} m^{a} t^{b} t^{c}
$$

We solve this condition by fixing the value of the dilaton $s$. We also impose positive intersection numbers and positive values for for the fluxes $m^{0}$ and $m^{a}$ so that if the $t^{a}$ are positive so is the dilaton. We also see that the dilaton scales like $t^{a}$. The real part of the superpotential imposes a condition on $e_{0}$ which fixes its value. Now, consider the real part of the condition $\partial_{S} W=0$ which gives

$$
e_{\tilde{a}} t^{\tilde{a}}+e_{\bar{a}} t^{\bar{a}}=0 .
$$

We solve this condition by fixing one of the fluxes $e_{\bar{a}}$. The imaginary part of the derivative gives

$$
h_{0}=\frac{1}{2} K_{a b c} m^{a} t^{b} t^{c}
$$

which we solve by fixing $h_{0}$. The imaginary parts of the conditions $\partial_{U_{k}} W=0$ impose that $h^{k}=0$. The real parts give the $p$ conditions

$$
e_{\tilde{a}}^{k} t^{\tilde{a}}+e_{\bar{a}}^{k} t^{\bar{a}}=0
$$

Since the matrix $e_{a}{ }^{k}$ has maximal rank, we can choose the square matrix $E_{1} \equiv e_{\tilde{a}}{ }^{k}$ to have non-vanishing determinant. Therefore, if we also define $E_{2} \equiv e_{\bar{a}}{ }^{k}$, we can solve the constraints (4.48) by taking

$$
t^{\tilde{a}}=-\left(E_{1}^{-1} E_{2}\right)_{\bar{a}}^{\tilde{a}} t^{\bar{a}} .
$$

We now impose the conditions on the fluxes that $-\left(E_{1}{ }^{-1} E_{2}\right)^{\tilde{a}}{ }_{\bar{a}}$ is positive. This does not fix the fluxes but does limit their possible values. It means that if the $t^{\bar{a}}$ are positive, so are $t^{\tilde{a}}$. For alter convenience we also impose $E_{1}=\left(E_{1}\right)^{T}$ which halves the degrees of freedom. Let us 
now focus on a solution in which $t^{\bar{a}}=\alpha m^{\bar{a}}$. The real constant $\alpha$ sets the scale of the moduli $t^{a}$ and $s$. The final set of conditions come from $\partial_{T^{a}} W=0$. These are $n$ constraints that read

$$
\frac{i}{2} m^{0} K_{a b c} T^{b} T^{c}+K_{a b c} p^{b} T^{c}-i q^{a}-i K_{a b c} S m^{b} T^{c}+e_{a} S+e_{a}{ }^{k} U_{k}=0 .
$$

Taking the real part of (4.50) gives

$$
K_{a b c} p^{b} t^{c}+\sigma K_{a b c} m^{b} t^{c}+s e_{a}+e_{a}^{k} u_{k}=0 .
$$

We now contract the last expression with $t^{a}$ which gives an equation that fixes $\sigma$ in terms of $t^{a}$

$$
\sigma=-\frac{K_{a b c} p^{a} t^{b} t^{c}}{K_{a b c} m^{a} t^{b} t^{c}}
$$

There remain $n-1$ constraints. The first $p$ of these fix the real parts of the complex moduli

$$
u_{k}=-\left(E_{1}^{-1}\right)_{k}^{\tilde{a}}\left[e_{\tilde{a}} s+K_{\tilde{a} b c} p^{b} t^{c}-K_{\tilde{a} b c} m^{b} t^{c} \frac{K_{b c d} p^{b} t^{c} t^{d}}{K_{b c d} m^{b} t^{c} t^{d}}\right]
$$

Later we take, $-\left(E_{1}^{-1}\right)_{k}^{\tilde{a}} e_{\tilde{a}} \gg 1$, in which case we see that the $u_{k}$ are positive. Putting the solutions (4.53) back into the remaining $(n-p-1)$ components of (4.51), we find $(n-p-1)$ constraints on the fluxes which we solve by fixing the remaining $e_{\bar{a}}$ fluxes (recall that one was fixed by (4.46) ). Similar arguments apply when taking the imaginary part of (4.50) which reads

$$
\frac{m^{0}}{6} K_{a b c} t^{b} t^{c}-q_{a}-\frac{s}{2} K_{a b c} m^{b} t^{c}+e_{a} \sigma+e_{a}{ }^{k} \nu_{k}=0
$$

The first $p$ of these conditions fix the axions $v_{k}$ and the remaining $(n-p)$ equations give constraints on the fluxes. We solve these by fixing the fluxes $q_{\bar{a}}$.

We have now solved all the supersymmetry equations and can apply the tadpole conditions to these solutions. The tadpole conditions read

$$
\begin{aligned}
m^{a} e_{a}^{k} & =0 . \\
-m^{0} h_{0}-p^{a} e_{a}-m^{a} q_{a} & =Q_{0}, \\
-p^{a} e_{a}^{k} & =Q^{k}
\end{aligned}
$$

The $p$ constraints (4.55) are solved by fixing $m^{\tilde{a}}$. We now set $p^{\bar{a}}=0$ which means that the $p$ conditions of (4.57) can be solved by taking

$$
p^{\tilde{a}}=-\left(E_{1}{ }^{-1}\right)^{\tilde{a}}{ }_{k} Q^{k} .
$$

We are left with the single tadpole equation (4.56). This tadpole fixes the overall scale factor $\alpha$, that is, the overall scale of the moduli. To see this consider contracting (4.54) with $t^{a}$, and using (4.45). One finds the relation

$$
q_{a} t^{a}=\frac{m^{0}}{6} K_{a b c} t^{a} t^{b} t^{c}=\frac{s}{2} K_{a b c} t^{a} m^{b} t^{c}
$$


Now we set $q^{\tilde{a}}=0$ which means that (4.59) gives

$$
q_{\bar{a}} m^{\bar{a}}=\alpha^{2} H_{1},
$$

where $H_{1}$ is a real number that depends on $K_{a b c}, e_{a}{ }^{k}, m^{\bar{a}}$ and $m^{0}$. Also (4.47) gives

$$
m^{0} h_{0}=\alpha^{2} H_{2}
$$

where $\mathrm{H}_{2}$ is a real number which depends on $K_{a b c}, e_{a}{ }^{k}, m^{a}$ and $m^{0}$. Therefore using (4.56) we can write

$$
\alpha^{2}=\frac{-Q_{0}+e_{\tilde{a}}\left(E_{1}^{-1}\right)_{k}^{\tilde{a}} Q^{k}}{H_{2}+H_{1}} .
$$

But this last formula ensures that, by taking $Q^{k}$ negative and $-\left(E_{1}{ }^{-1}\right)_{k}{ }^{\tilde{a}} e_{\tilde{a}} \gg 1$, one can find parametrically large values for $\alpha$ and so for all the moduli.

\subsection{Ten-dimensional uplifts and torsion classes}

So far we have considered type II theories on non-geometric backgrounds with $S U(3) \times S U(3)$ structure and we have studied solutions of the four-dimensional truncations thereof. However, until now we have not studied whether such backgrounds are consistent string backgrounds or if the solutions obtained are indeed solutions of the full ten-dimensional string/supergravity theories. In this section we precisely want to fill this gap.

A simple argument to show the consistency of our solutions with the ten-dimensional picture is to note that the superpotential of [30], which we generalised in our paper, was derived from the reduction of the fermionic action. Therefore, the solution to the four-dimensional supersymmetry equations derived from such a superpotential will solve the ten-dimensional supersymmetry variations as well.

For the case at hand this can also be seen explicitely as follows. The conditions for $N=1$ Minkowski vacua, which spelled out in [4] (see also [3]), require that the internal manifold is a twisted generalised Calabi-Yau manifold defined by the condition

$$
d_{H} \Pi^{e v}=0
$$

provided we are in a regime where the dilaton and the warp factor are constant over the internal manifold.

As we argued before in the paper, for the non-geometric backgrounds we consider one has to replace the twisted exterior derivative $d_{H}$ by the covariant derivative of (2.22). Therefore, for our case the condition for $N=1$ Minkowski vacua reads

$$
\mathcal{D} \Pi^{e v}=0
$$

It is possible to see then that the above equation is equivalent to the supersymmetry conditions corresponding to the superpotential (3.29) along the directions $S$ and $U^{k}$. This implies that in a supersymmetric Minkowski vacuum the moduli take values such that the manifold is twisted (non-geometric) generalised Calabi-Yau as required by the ten-dimensional analysis. 


\section{Summary}

In this paper we studied compactifications of type IIA string theory on manifolds with $S U(3) \times$ $S U(3)$ structure in the presence of $O 6$-planes and its mirror compactification of type IIB on manifolds with $S U(3) \times S U(3)$ structure in the presence of $O 3$-planes. We argued that generalised geometry provides us with the tools needed to treat these non-geometric compactifications in a geometric sense. By introducing a covariant derivative for T-dualities we were able to derive the four-dimensional superpotential. We showed that, in the presence of non-geometric fluxes, if the number (in IIA notation) of Kähler moduli is larger than the number of complex structure moduli, the theory contains supersymmetric Minkowski vacua with all the moduli stabilised in a a perturbative regime. We find that if there are no complex structure moduli then the value of the moduli in the vacuum is capped by the orientifold charge. In the presence of complex structure moduli however there are parametrically controlled vacua.

The possible extensions to this work are numerous, and we hope that the systematic construction of Minkowski vacua presented in this paper will help eliminate the need to uplift the usual anti deSitter vacua to Minkowski, and all the problems associated with this mechanism such as fine tuning and high scale supersymmetry breaking.

\section{Acknowledgments}

The authors would like to thank Gil Cavalcanti, James Gray, Thomas Grimm, Andre Lukas and David Skinner for useful discussions.

EP is supported by a PPARC Postdoctoral Fellowship. GT and AM were partially supported by the EC $6^{\text {th }}$ Framework Programme MRTN-CT-2004-503369, and by the EU 6th Framework Marie Curie Research and Training network" (MRTN-CT-2006-035863) "UniverseNet". AM was also supported by the European project MRTN-CT-2004-005104 "ForcesUniverse" and SFB-Transregio 33 "The Dark Universe" by Deutsche Forschungsgemeinschaft (DFG).

\section{Appendix}

\section{A Full solution with two Kähler moduli}

In this appendix we include for completeness the rest of the two Kähler moduli case studied in section 4.2.2. We also include a set of flux parameters as an explicit solution. The axions read

$$
\begin{aligned}
b^{1}= & b^{2}=0, \\
\sigma= & \frac{e_{4}}{e_{3} m^{1}}\left(p^{2}+\frac{Q_{1}\left(e_{4}{ }^{2} k_{0}-2 e_{3} e_{4} k_{1}+e_{3}{ }^{2} k_{2}\right)}{F_{1}}\right), \\
\nu= & \frac{-1}{4 e_{3} e_{4} F_{1} m^{1}}\left[4 e_{2} e_{4} F_{1} p^{2}-4 e_{3} F_{1} m^{1} q_{2}-e_{3} e_{4}\left(e_{4}{ }^{2} k_{1}-2 e_{3} e_{4} k_{2}+e_{3}{ }^{2} k_{3}\right) Q_{0}\right. \\
& \left.+e_{2}\left(4 e_{4}{ }^{3} k_{0}-7 e_{3} e_{4}{ }^{2} k_{1}+2 e_{3}{ }^{2} e_{4} k_{2}+e_{3}{ }^{3} k_{3}\right) Q_{1}\right] .
\end{aligned}
$$


The fixed fluxes are

$$
\begin{aligned}
m^{2} & =-m^{1} \frac{e_{3}}{e_{4}} \\
e_{1} & =\frac{e_{2} e_{3}}{e_{4}} \\
p^{1} & =-\frac{e_{4} p^{2}+Q_{1}}{e_{3}}, \\
q_{1} & =\frac{1}{m^{1}}\left(-Q_{0}+\frac{q_{2} m^{1} e_{3}}{e_{4}}-p^{1} e_{1}-p^{2} e_{2}-m^{0} h_{0}\right), \\
h_{0} & =\frac{3\left(-e_{4} Q_{0}+e_{2} Q_{1}\right)}{4 e_{4} m^{0}}, \\
h_{1} & \left.=0, \quad\left(p^{2} F_{1}+Q_{1}\left(e_{4}{ }^{2} k_{0}-2 e_{3} e_{4} k_{1}+e_{3}{ }^{2} k_{2}\right)\right)\right] . \\
e_{0} & =-\frac{3}{4 e_{3} F_{1}{ }^{2} m^{0} m^{1}}\left[\left(e_{4}{ }^{3} k_{0}-4 e_{3} e_{4}{ }^{2} k_{1}+4 e_{3}{ }^{2} e_{4} k_{2}-e_{3}{ }^{3} k_{3}\right)\left(e_{4} Q_{0}-e_{2} Q_{1}\right)\right.
\end{aligned}
$$

An explicit example of a solution for the intersection numbers (4.36) is presented in table 2

\begin{tabular}{||c|c|c|c|c|c|c|c|c|c|c|c|c|c|c|c|c|c||}
\hline$v^{1}$ & $v^{2}$ & $s$ & $u$ & $m^{0}$ & $m^{1}$ & $m^{2}$ & $e_{0}$ & $e_{1}$ & $e_{2}$ & $e_{3}$ & $e_{4}$ & $p^{1}$ & $p^{2}$ & $q_{1}$ & $q_{2}$ & $h_{0}$ & $h_{1}$ \\
\hline 4.6 & 4.6 & 6.1 & 154 & 4 & 1 & 1 & 432 & 94 & -94 & -4 & 4 & -8 & 0 & 196 & 0 & 147 & 0 \\
\hline
\end{tabular}

Table 2: Table showing values of flux parameters and moduli values for an explicit solution.

\section{References}

[1] M. Grana, "Flux compactifications in string theory: A comprehensive review," Phys. Rept. 423 (2006) 91 arXiv:hep-th/0509003.

[2] M. R. Douglas and S. Kachru, "Flux compactification," arXiv:hep-th/0610102.

[3] M. Grana, R. Minasian, M. Petrini and A. Tomasiello, "Supersymmetric backgrounds from generalized Calabi-Yau manifolds," JHEP 0408 (2004) 046 arXiv:hep-th/0406137.

[4] M. Grana, R. Minasian, M. Petrini and A. Tomasiello, "Generalized structures of N = 1 vacua," JHEP 0511 (2005) 020 arXiv:hep-th/0505212.

[5] K. Becker, M. Becker, C. Vafa and J. Walcher, "Moduli stabilization in non-geometric backgrounds," arXiv:hep-th/0611001.

[6] D. Krefl and D. Lust, "On supersymmetric Minkowski vacua in IIB orientifolds," JHEP 0606, 023 (2006) arXiv:hep-th/0603166.

[7] S. Gurrieri, J. Louis, A. Micu and D. Waldram, "Mirror symmetry in generalized Calabi-Yau compactifications," Nucl. Phys. B 654, 61 (2003) arXiv:hep-th/0211102.

[8] S. Kachru, M. B. Schulz, P. K. Tripathy and S. P. Trivedi, "New supersymmetric string compactifications," JHEP 0303, 061 (2003) arXiv:hep-th/0211182. 
[9] S. Gurrieri and A. Micu, "Type IIB theory on half-flat manifolds," Class. Quant. Grav. 20, 2181 (2003) arXiv:hep-th/0212278.

[10] J. P. Derendinger, C. Kounnas, P. M. Petropoulos and F. Zwirner, "Superpotentials in IIA compactifications with general fluxes," Nucl. Phys. B 715, 211 (2005) arXiv:hep-th/0411276.

[11] G. Villadoro and F. Zwirner, " $\mathrm{N}=1$ effective potential from dual type-IIA D6/O6 orientifolds with general fluxes," JHEP 0506, 047 (2005) arXiv:hep-th/0503169.

[12] T. House and E. Palti, "Effective action of (massive) IIA on manifolds with SU(3) structure," Phys. Rev. D 72, 026004 (2005) arXiv:hep-th/0505177.

[13] P. G. Camara, A. Font and L. E. Ibanez, "Fluxes, moduli fixing and MSSM-like vacua in a simple IIA orientifold," JHEP 0509, 013 (2005) arXiv:hep-th/0506066.

[14] M. Grana, J. Louis and D. Waldram, "Hitchin functionals in N = 2 supergravity," JHEP 0601, 008 (2006) arXiv:hep-th/0505264.

[15] B. S. Acharya, F. Benini and R. Valandro, "Fixing moduli in exact type IIA flux vacua," arXiv:hep-th/0607223

[16] G. Curio and A. Krause, "Four-flux and warped heterotic M-theory compactifications," Nucl. Phys. B 602, 172 (2001) arXiv:hep-th/0012152.

[17] K. Becker and K. Dasgupta, "Heterotic strings with torsion," JHEP 0211, 006 (2002) arXiv:hep-th/0209077.

[18] G. Curio and A. Krause, "Enlarging the parameter space of heterotic M-theory flux compactifications to phenomenological viability," Nucl. Phys. B 693, 195 (2004) arXiv:hep-th/0308202.

[19] A. Micu, E. Palti and P. M. Saffin, "M-theory on seven-dimensional manifolds with SU(3) structure," JHEP 0605, 048 (2006) arXiv:hep-th/0602163.

[20] S. Gurrieri, A. Lukas and A. Micu, "Heterotic on half-flat," Phys. Rev. D 70, 126009 (2004) arXiv:hep-th/0408121.

[21] A. Micu, "Heterotic compactifications and nearly-Kaehler manifolds," Phys. Rev. D 70, 126002 (2004) arXiv:hep-th/0409008.

[22] R. D'Auria, S. Ferrara, M. Trigiante and S. Vaula, "Gauging the Heisenberg algebra of special quaternionic manifolds," Phys. Lett. B 610 (2005) 147 arXiv:hep-th/0410290.

[23] R. D'Auria, S. Ferrara, M. Trigiante and S. Vaula, "Scalar potential for the gauged Heisenberg algebra and a non-polynomial antisymmetric tensor theory," Phys. Lett. B 610 (2005) 270 arXiv:hep-th/0412063.

[24] B. de Carlos, S. Gurrieri, A. Lukas and A. Micu, "Moduli stabilisation in heterotic string compactifications," JHEP 0603, 005 (2006) arXiv:hep-th/0507173.

[25] P. Manousselis, N. Prezas and G. Zoupanos, "Supersymmetric compactifications of heterotic strings with fluxes and condensates," Nucl. Phys. B 739, 85 (2006) arXiv:hep-th/0511122.

[26] L. Anguelova and K. Zoubos, "Flux superpotential in heterotic M-theory," Phys. Rev. D 74, 026005 (2006) arXiv:hep-th/0602039. 
[27] K. Behrndt, M. Cvetic and P. Gao, "General type IIB fluxes with SU(3) structures," Nucl. Phys. B 721, 287 (2005) arXiv:hep-th/0502154.

[28] E. Palti, "Aspects of moduli stabilisation in string and M-theory," arXiv:hep-th/0608033.

[29] M. Grana, "Flux Compactifications And Generalized Geometries," Class. Quant. Grav. 23, S883 (2006).

[30] I. Benmachiche and T. W. Grimm, "Generalized N = 1 orientifold compactifications and the Hitchin functionals," Nucl. Phys. B 748, 200 (2006) arXiv:hep-th/0602241.

[31] M. Grana, J. Louis and D. Waldram, "SU(3) x SU(3) compactification and mirror duals of magnetic fluxes," arXiv:hep-th/0612237.

[32] S. Hellerman, J. McGreevy and B. Williams, "Geometric constructions of nongeometric string theories," JHEP 0401, 024 (2004) arXiv:hep-th/0208174.

[33] A. Dabholkar and C. Hull, "Duality twists, orbifolds, and fluxes," JHEP 0309, 054 (2003) arXiv:hep-th/0210209.

[34] D. A. Lowe, H. Nastase and S. Ramgoolam, "Massive IIA string theory and matrix theory compactification," Nucl. Phys. B 667, 55 (2003) arXiv:hep-th/0303173.

[35] A. Flournoy, B. Wecht and B. Williams, "Constructing nongeometric vacua in string theory," Nucl. Phys. B 706, 127 (2005) arXiv:hep-th/0404217.

[36] V. Mathai and J. M. Rosenberg, "On mysteriously missing T-duals, H-flux and the T-duality group," arXiv:hep-th/0409073.

[37] V. Mathai and J. M. Rosenberg, "T-duality for torus bundles via noncommutative topology," Commun. Math. Phys. 253, 705 (2004) arXiv:hep-th/0401168].

[38] P. Bouwknegt, K. Hannabuss and V. Mathai, "Nonassociative tori and applications to T-duality," Commun. Math. Phys. 264, 41 (2006) arXiv:hep-th/0412092.

[39] C. M. Hull, "A geometry for non-geometric string backgrounds," JHEP 0510, 065 (2005) arXiv:hep-th/0406102.

[40] C. M. Hull and R. A. Reid-Edwards, "Flux compactifications of string theory on twisted tori," arXiv:hep-th/0503114.

[41] J. Shelton, W. Taylor and B. Wecht, "Nongeometric flux compactifications," JHEP 0510 (2005) 085 arXiv:hep-th/0508133.

[42] L. Martucci and P. Smyth, "Supersymmetric D-branes and calibrations on general N = 1 backgrounds," JHEP 0511 (2005) 048 arXiv:hep-th/0507099.

[43] J. Gray and E. J. Hackett-Jones, "On T-folds, G-structures and supersymmetry," JHEP 0605, 071 (2006) arXiv:hep-th/0506092.

[44] A. Dabholkar and C. Hull, "Generalised T-duality and non-geometric backgrounds," JHEP 0605, 009 (2006) arXiv:hep-th/0512005.

[45] A. Lawrence, M. B. Schulz and B. Wecht, "D-branes in nongeometric backgrounds," JHEP 0607, 038 (2006) arXiv:hep-th/0602025. 
[46] C. M. Hull and R. A. Reid-Edwards, "Flux compactifications of M-theory on twisted tori," JHEP 0610, 086 (2006) arXiv:hep-th/0603094.

[47] C. M. Hull, "Global aspects of T-duality, gauged sigma models and T-folds," arXiv:hep-th/0604178.

[48] C. M. Hull, "Doubled geometry and T-folds," arXiv:hep-th/0605149

[49] J. Shelton, W. Taylor and B. Wecht, "Generalized flux vacua," arXiv:hep-th/0607015

[50] I. Ellwood and A. Hashimoto, "Effective descriptions of branes on non-geometric tori," arXiv:hep-th/0607135.

[51] P. Grange and S. Schafer-Nameki, "T-duality with H-flux: Non-commutativity, T-folds and G x G structure," arXiv:hep-th/0609084.

[52] I. Ellwood, "NS-NS fluxes in Hitchin's generalized geometry," arXiv:hep-th/0612100

[53] A. Kapustin and Y. Li, "Topological sigma-models with H-flux and twisted generalized complex arXiv:hep-th/0407249.

[54] J. Persson, "T-duality and generalized complex geometry," arXiv:hep-th/0612034

[55] G. Aldazabal, P. G. Camara, A. Font and L. E. Ibanez, "More dual fluxes and moduli fixing," JHEP 0605, 070 (2006) arXiv:hep-th/0602089.

[56] N. Hitchin, "Generalized Calabi-Yau manifolds," Quart. J. Math. Oxford Ser. 54 (2003) 281 arXiv:math.dg/0209099.

[57] N. Hitchin, "Instantons, Poisson structures and generalized Kaehler geometry," Commun. Math. Phys. 265 (2006) 131 arXiv:math.dg/0503432.

[58] N. Hitchin, "Brackets, forms and invariant functionals," arXiv:math.dg/0508618.

[59] S. Chiossi and S. Salamon, "The Intrinsic Torsion of $S U(3)$ and $G_{2}$ Structures," in Differential geometry, Valencia, 2001, pp. 115, arXiv: math.DG/0202282

[60] M. Gualtieri, "Generalized Complex Geometry," Oxford University DPhil thesis (2004) arXiv:math.DG/0401221].

[61] G. R. Cavalcanti, "New aspects of the ddc-lemma," Oxford University DPhil thesis (2004) arXiv:math.dg/0501406.

[62] F. Witt, "Generalised $G_{2}$-manifolds," Commun. Math. Phys. 265 (2006) 275 arXiv:math.dg/0411642;

F. Witt, "Special metric structures and closed forms", Oxford University DPhil thesis (2004) arXiv:math.DG/0502443.

[63] M. Grana, R. Minasian, M. Petrini and A. Tomasiello, "A scan for new N = 1 vacua on twisted tori," arXiv:hep-th/0609124.

[64] A. Kapustin, "Topological strings on noncommutative manifolds," Int. J. Geom. Meth. Mod. Phys. 1, 49 (2004) arXiv:hep-th/0310057.

[65] A. K. Kashani-Poor and R. Minasian, "Towards reduction of type II theories on SU(3) structure manifolds," arXiv:hep-th/0611106. 
[66] J. Louis and A. Micu, "Heterotic-type IIA duality with fluxes," arXiv:hep-th/0608171.

[67] K. Behrndt and M. Cvetic, "General N = 1 supersymmetric fluxes in massive type IIA string theory," Nucl. Phys. B 708 (2005) 45 arXiv:hep-th/0407263.

[68] O. DeWolfe, A. Giryavets, S. Kachru and W. Taylor, "Type IIA moduli stabilization," JHEP 0507, 066 (2005) arXiv:hep-th/0505160. 